\title{
Macrocyclic oligomers as compatibilizing agent for hemp fibres/biodegradable polyester eco-composites
}

DOI:

10.1016/j.polymer.2018.05.053

\section{Document Version}

Accepted author manuscript

Link to publication record in Manchester Research Explorer

\section{Citation for published version (APA):}

Conzatti, L., Brunengo, E., Utzeri, R., Castellano, M., Hodge, P., \& Stagnaro, P. (2018). Macrocyclic oligomers as compatibilizing agent for hemp fibres/biodegradable polyester eco-composites. Polymer, 146, 396-406. https://doi.org/10.1016/j.polymer.2018.05.053

\section{Published in:}

Polymer

\section{Citing this paper}

Please note that where the full-text provided on Manchester Research Explorer is the Author Accepted Manuscript or Proof version this may differ from the final Published version. If citing, it is advised that you check and use the publisher's definitive version.

\section{General rights}

Copyright and moral rights for the publications made accessible in the Research Explorer are retained by the authors and/or other copyright owners and it is a condition of accessing publications that users recognise and abide by the legal requirements associated with these rights.

\section{Takedown policy}

If you believe that this document breaches copyright please refer to the University of Manchester's Takedown Procedures [http://man.ac.uk/04Y6Bo] or contact uml.scholarlycommunications@manchester.ac.uk providing relevant details, so we can investigate your claim.

\section{OPEN ACCESS}


Elsevier Editorial System(tm) for Polymer Manuscript Draft

Manuscript Number: POLYMER-18-340R1

Title: Macrocyclic oligomers as compatibilizing agent for hemp fibres/biodegradable polyester eco-composites

Article Type: Research Paper

Section/Category: Polymer Synthesis

Keywords: entropically-driven ring-opening polymerization (ED-ROP) ; macrocyclic oligomers (MCOs); hemp fibres eco-composites

Corresponding Author: Dr. Lucia Conzatti, Ph. D.

Corresponding Author's Institution: Consiglio Nazionale delle Ricerche

First Author: Lucia Conzatti, Ph. D.

Order of Authors: Lucia Conzatti, Ph. D.; Elisabetta Brunengo; Roberto Utzeri; Maila Castellano; Philip Hodge; Paola Stagnaro

Abstract: Ring:chain equilibria (RCE), existing under appropriate reaction conditions, between a linear polyester and the corresponding family of macrocyclic oligomers (MCOs), were exploited to develop an original compatibilizing pathway for preparing eco-composites based on hemp fibres and a biodegradable random co-polyester, namely poly (1,4-butylene adipate-co-terephthalate) (PBAT) with 50:50 adipate:therephthalate proportion. Cyclo-depolymerization (CDP) of PBAT was successfully carried out at high dilution using various solvents, catalysts, and reaction times. The reconversion of MCOs into linear chains by entropically-driven ring-opening polymerization (ED-ROP) ocCurs by simple heating in the bulk. This reaction, after optimization of catalyst amount, temperature, and reaction time, was used for the surface modification of hemp fibres. PBAT-based eco-composites containing hemp fibres as such or pre-modified by treatment with the MCOs were then prepared and characterized by morphological and mechanical analysis. 


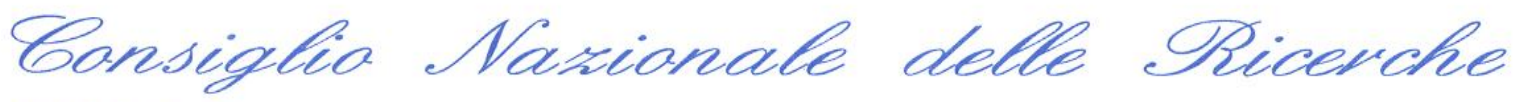

\section{ISMAC - ISTITUTO PER LO STUDIO DELLE MACROMOLECOLE}

GENOVA

Via De Marini, 6 - 16149 GENOVA Italy - Tel. (39) 0106475879 - Fax (39) 0106475880

Codice fiscale 80054330586 - Partita Iva 02118311006

Dear Editor,

I am sending the revised version of the manuscript POLYMER-18-340 "Macrocyclic oligomers as compatibilizing agent for hemp fibres/biodegradable polyester eco-composites" by L. Conzatti, E. Brunengo, R. Utzeri, M. Castellano, P. Hodge, and P. Stagnaro.

It has been revised according to the referees' comments: the whole text was improved whenever needed and the results obtained by thermogravimetric an dynamic-mechanical analysis of the ecocomposites were added. All the revisions done are evidenced in red color.

We have answered all the remarks, as you can see in the attached "Response to Reviewers" file.

We warmly thank the referees for the suggestions aimed at improving the paper.

On behalf of all the authors, I warmly hope the paper can now deserve consideration for publication in Polymer journal.

Thank you in advance for your kind attention. Looking forward to hearing from you.

Sincerely yours,

Lucia Conzatti

Genova, May $11^{\text {th }}, 2018$ 
Ms. No. POLYMER-18-340

"Macrocyclic oligomers as compatibilizing agent for hemp fibres/biodegradable polyester eco-
composites"

\section{Response to Reviewers}

Point-by-point the response to Reviewers is given in red in the following.

\section{Reviewer \#1 (Major Revision):}

This work is very interesting in line with the current trends in biodegradable plastics and natural reinforcing fillers. The modification part is novel and the results are thoroughly presented.

We warmly appreciate the positive comments of the Reviewer on the work in the whole.

However, there some modification which will make this article much more useful to the readers.

1. Thermal properties of the composites may be added.

Experimental setup used thermogravimetric analysis (TGA) of the composites, results obtained from and related comments were added in the Manuscript at Pag. 7 - Lines 200-204, at Pag. 16 - Lines 372-379, and at Pag. 20-21 - Lines 464-476.

For homogeneity purposes, also TGA data under $\mathrm{N}_{2}$ atmosphere of the fibres were reported in Table 3 of the revised Manuscript (Table 3, Pag. 17 - Lines 381-383).

2. The tensile strain decreased in the sample containing the MCO-modified fibres, how the explain effectiveness of the system to be at best at this composition as normally tensile strain is used to indicate the compatibility between matrix-filler.

To answer to this point, the following sentence was added at Pag. 21-22 - Lines 490-495:

"The slight decrease of the stress at break could be ascribed to a mechanical weakening of the hemp fibres due to the thermal treatment carried out during the ED-ROP reaction $\left(190^{\circ} \mathrm{C}, 30 \mathrm{~min}\right)$. Even though a TGA isothermal analysis carried out at $200^{\circ} \mathrm{C}$ in $\mathrm{O}_{2}$ atmosphere for $30 \mathrm{~min}$ revealed a good thermo-oxidative resistance of $\mathrm{HF}_{\mathrm{Na}} \mathrm{S}$ fibres (the weight loss is about $0.5 \%$ ), the maintenance at high temperature in oxidative atmosphere could be slightly detrimental to the mechanical properties of the fibres, as reported by Prasad et al. [34]."

Moreover, in order to further support the enhanced fibre-PBAT adhesion/interaction due to the presence of MCOs, DMA tests were also carried out. Related data and comments were added in the revised Manuscript at Pag. 7 - Lines 208-210 and 217-223, and at Pag. 22 - Lines 496-515.

3. The composite at various compositions may be prepared and characterized for their mechanical properties.

The study of composites at various fibre composition is out of the scope of the present Manuscript, which is that of demonstrate the upcycling of HF residues on evaluating the efficiency of MCO as compatibilizing agent in PBAT-based eco-composites. For this purpose, such a high amount (about 40 wt.-\%) of HF was added to the PBAT matrix.

The study of eco-composites with different amounts of fibres will be the object of a future work. 


\section{Reviewer \#2 (Reject):}

\section{The reaction formula of $M C O$ should be added.}

The scheme of the ring:chain equilibrium of MCOs vs. PBAT was added (Scheme 2 ) in the text at Pag. $3-$ Lines 85-86.

\section{Why did the authors choose such a polyester with so wide PDI, about 8?}

This polyester grade was suggested from BASF technical personnel as the most suitable for the preparation of eco-composites by melt blending technique.

3.In page 11, "the low melting range confirms the considerations on the relative volatility of the MCOs previously done". What's the relationship between the melting point and the volatility of MCOs? The DSC and TGA curves of MCOs are recommended to be added in the supporting information.

We agree with the referee: the relationship between the melting point and the volatility of MCOs is not directly proportional. However, DSC heating curve of MCOs shows a broad endothermic peak ranging from 30 to $60^{\circ} \mathrm{C}$ indicating a very low melting point. The relative volatility of MCOs could be enhanced in the conditions used for the removal of the $o-D C B$ solvent, during which they were maintained at relatively high temperature $\left(70^{\circ} \mathrm{C}\right)$ under vacuum. Moreover, it is to be outlined that the most volatile one is expected to be the monomeric cyclic n-butyl adipate.

A sentence was added at Pag. 9 - Lines 258-259 and that at Pag. 11 - Lines 288-289 was properly modified as "(this low melting range supports the considerations on the relative volatility of the MCOs previously done)".

Actually, DSC analyses were carried out, even though the related curves are not shown because they lack of significance in the Manuscript context. In facts, ED-ROP of MCOs takes place during the DSC (or TGA) heating run resulting in a dynamic system which leads to the PBAT formation.

4.In page 19, "HFNas, that only exhibits a feeble signal ascribable to carbonyl groups centred at $1735 \mathrm{~cm}-1 . "$ However, as shown in Figure 9, there is no peak at 1725 for the curve of HFNas, but there is a peak at around 1690.

The authors thank the referee for the suggestion, Figure 9 and relative caption (Pag. 18 - Lines 414-418) were improved inserting an inset to help the readers.

5.In page 22, although the storage modulus (E) of PBAT + HFNa-npMCOs is larger than that of PBAT + HFs, the stress at break (<sigma $>$ b) and the elongation at break (<xi>) are lower obviously. Why?

To answer to this point, the following sentence was added at Pag. 21-22 - Lines 490-495:

"The slight decrease of the stress at break could be ascribed to a mechanical weakening of the hemp fibres due to the thermal treatment carried out during the ED-ROP reaction $\left(190^{\circ} \mathrm{C}, 30 \mathrm{~min}\right)$. Even though a TGA isothermal analysis carried out at $200^{\circ} \mathrm{C}$ in $\mathrm{O}_{2}$ atmosphere for $30 \mathrm{~min}$ revealed a good thermo-oxidative resistance of $\mathrm{HF}_{\mathrm{Na}} \mathrm{S}$ fibres (the weight loss is about $0.5 \%$ ), the maintenance at high temperature in oxidative atmosphere could be slightly detrimental to the mechanical properties of the fibres, as reported by Prasad et al. [34]."

Moreover, in order to further support the enhanced fibre-PBAT adhesion/interaction due to the presence of MCOs, DMA tests were also carried out. Related data and comments were added in the revised Manuscript at Pag. 7 - Lines 208-210 and 217-223, and at Pag. 22 - Lines 496-515. 

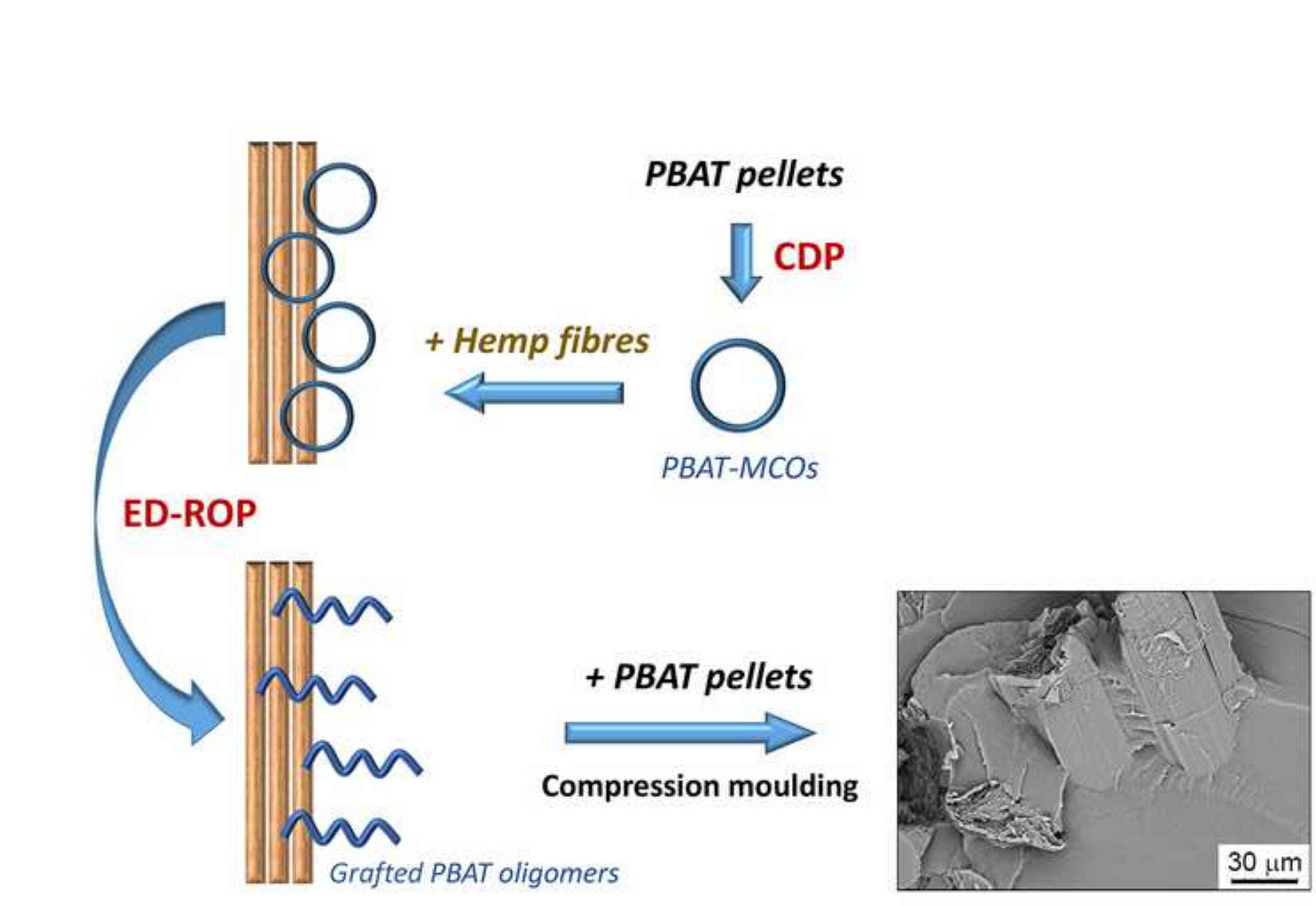

ED-ROP

+ PBAT pellets

Compression moulding
Hemp fibres

\section{PBAT-MCOS}

\section{$\sqrt{ } \mathrm{CDP}$}


1 Macrocyclic oligomers as compatibilizing agent for hemp fibres/biodegradable polyester eco-composites

\author{
Lucia Conzatti $^{1 *}$, Elisabetta Brunengo ${ }^{1}$, Roberto Utzeri ${ }^{1}$, Maila Castellano ${ }^{2}$, Philip Hodge ${ }^{3}$, \\ Paola Stagnaro ${ }^{1}$
}

${ }^{1}$ Istituto per lo Studio delle Macromolecole (ISMAC) - Sede di Genova, CNR, Via De Marini 6, 16149 Genova, Italy

${ }^{2}$ Dipartimento di Chimica e Chimica Industriale, Università di Genova, Via Dodecaneso 31, 16146 Genova, Italy

${ }^{3}$ Department of Chemistry, University of Manchester, Oxford Road, Manchester M13 9PL, UK

*Corresponding author: Tel.: +39 0106475866; Fax: +39 0106475880. E-mail address: lucia.conzatti@ge.ismac.cnr.it (L.Conzatti)

\title{
Abstract
}

Ring:chain equilibria (RCE), existing under appropriate reaction conditions, between a linear polyester and the corresponding family of macrocyclic oligomers (MCOs), were exploited to develop an original compatibilizing pathway for preparing eco-composites based on hemp fibres and a biodegradable random co-polyester, namely poly(1,4-butylene adipate-co-terephthalate) (PBAT) with 50:50 adipate:therephthalate proportion.

Cyclo-depolymerization (CDP) of PBAT was successfully carried out at high dilution using various solvents, catalysts, and reaction times. The reconversion of MCOs into linear chains by entropically-driven ring-opening polymerization (ED-ROP) occurs by simple heating in the bulk. This reaction, after optimization of catalyst amount, temperature, and reaction time, was used for the surface modification of hemp fibres. PBAT-based eco-composites containing hemp fibres as such or pre-modified by treatment with the MCOs were then prepared and characterized by morphological and mechanical analysis.

Keywords: entropically-driven ring-opening polymerization (ED-ROP); macrocyclic oligomers (MCOs); hemp fibres; biodegradable polyester; eco-composites; mechanical properties. 


\section{Introduction}

36 In the last decade, macrocyclic oligomers (MCOs) and their entropically-driven ring-opening polymerization (ED-ROP) have become of interest for the synthesis of polycondensation polymers such as polyesters, polycarbonates, certain polyamides, and various high-performance aromatic polymers [1-3]. ED-ROPs are based on ring:chain equilibria (RCE), i.e. the well-known equilibria existing, under appropriate reaction conditions, between a condensation polymer and the corresponding family of MCOs (Scheme 1) $[1,2,4]$.

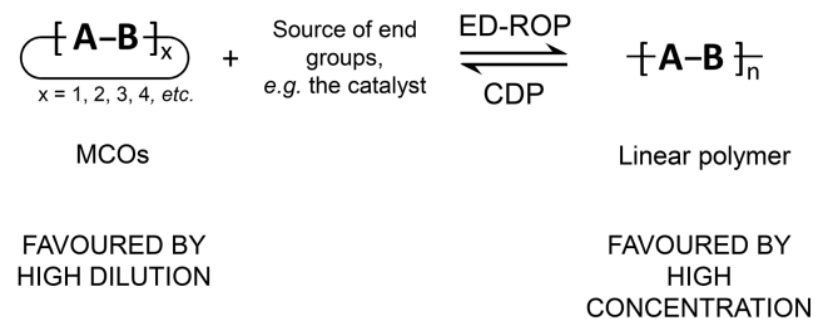

Scheme 1. A generalized ring:chain equilibrium. (1-column fitting image)

ED-ROP can be considered as a green process because to push the equilibrium towards the linear polymer generally no solvent is required, and, since the starting MCOs have no end groups and are strainless, no small molecules are released and little or no heat is evolved. Furthermore, highmolecular-weight polymers can be prepared in relatively short reaction times under atmospheric pressure, and the lower melt viscosity of the MCOs with respect to the polymer being formed allows easier processing by various techniques, such as pultrusion, resin-transfer moulding, reaction injection moulding, either concurrent with or just prior to polymerization [5-7]. ED-ROP has potential application in the preparation of composite materials [3], as voids due to the release of small volatile compounds are not created in the final products and the control of temperature throughout the reaction course is relatively facile. In a wider context, growing global environmental and social concerns, high rate of depletion of petroleum resources, and new environmental regulations have encouraged the search for green processes, and in this respect, ED-ROP of strainless MCOs appears perfectly suited [1,2]. Furthermore, the interest for new materials derived from renewable sources as well as for recycle and valorization of low cost, waste or difficult-to-dispose natural products, such as wool or lignocellulosic fibres obtained from the stem of hemp, flax, jute, bamboo, etc. [8-12] is continuously increasing. In this field, many scientific research projects, as well as many commercial programs, are nowadays focused on eco-composites, i.e. composite materials, based on natural fibres embedded in natural or biodegradable polymer matrices $[9,11]$, that can be easily degraded or bio- 
assimilated, thus possessing environmental and ecological advantages over conventional composites. In particular, eco-composite materials containing ligno-cellulosic natural fibres (hemp, flax, jute, etc.) are characterized by low density, low environmental impact, recyclability and favourable cost/performance ratio $[8,10,12]$.

Ligno-cellulosic fibres, which mainly consist of cellulose filaments embedded in a matrix of hemicellulose and lignin, have pronounced hydrophilic characteristics. This generally leads to a poor fibre-polymer interaction since the polymer matrix is typically apolar or only weakly polar. As a consequence, the attainment of eco-composites with good mechanical properties requires improvement in the compatibility between the two components $[8,10,12,13]$.

Within this framework, in recent years we have started a study aimed at improving the adhesion between natural fibres (in particular wool) and various polymer matrices [14-17], exploring different compatibilization strategies.

In the present work the ED-ROP of the MCOs, obtained by cyclo-depolymerization (CDP) of a commercial biodegradable random co-polyester, namely poly(1,4-butylene adipate-co-terephthalate) (PBAT) (50:50 adipate:therephthalate), was exploited to develop a novel compatibilization pathway for achieving eco-composites based on PBAT and hemp fibres (HFs). As a matter of fact, the MCOs, previously adsorbed onto the hemp fibres and converted into oligomeric chains by ED-ROP (Scheme 2), can act as a compatibilizing agent capable, at least in principle, of interacting with both the fibre surface and the polyester matrix directly during melt mixing and/or by proper thermal treatments.

MCOs

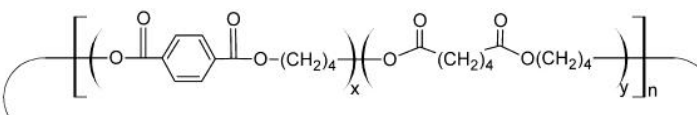

cat.

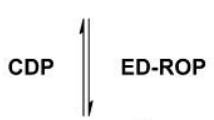

PBAT

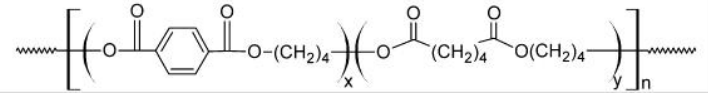

Scheme 2. Ring:chain equilibrium of PBAT. (1-column fitting image)

To this purpose, CDP of PBAT polyester was investigated at high dilution by varying the solvent, the catalyst and/or the reaction time. Selected MCOs were then used to optimize the conditions of the ED-ROP and then pre-adsorbed onto HFs previously mildly treated with aqueous $\mathrm{NaOH}$. Once the ED-ROP of the MCOs adsorbed onto HFs has taken place, the uncut fibres were combed and incorporated into the PBAT matrix by compression moulding. The ensuing eco-composites were 
then morphologically characterized to evaluate the fibre-matrix interaction and subjected to preliminary uniaxial tensile tests to investigate their mechanical properties.

\section{Materials and methods}

\subsection{Materials}

A commercial biodegradable, statistical poly(1,4-butylene adipate-co-terephthalate) (PBAT) grade (Ecoflex ${ }^{\circledR}$ F Blend A1200), kindly supplied by BASF (Germany), was used as the polyester matrix. $\bar{M}_{n}=11.7 \cdot 10^{3} \mathrm{~g} / \mathrm{mol}$ and $\bar{M}_{w}=84.4 \cdot 10^{3} \mathrm{~g} / \mathrm{mol}$ were evaluated by size exclusion chromatography (SEC). Values of $T_{\mathrm{g}}=-33^{\circ} \mathrm{C}, T_{\mathrm{m}}=118^{\circ} \mathrm{C}, \Delta H_{\mathrm{m}}=26 \mathrm{~J} / \mathrm{g}, T_{\mathrm{c}}=30^{\circ} \mathrm{C}$ and $\Delta H_{\mathrm{c}}=24$ $\mathrm{J} / \mathrm{g}$ were determined by differential scanning calorimetry (DSC). See the paragraph 2.6 for details on experimental conditions. The proportion of adipate versus terephthalate units was about $1: 1$, as determined by ${ }^{1} \mathrm{H}$ NMR analysis.

Raw hemp fibres of Carmagnola type, from which shives had been previously removed, were kindly supplied by Assocanapa (Italy) and used as received (HFs) or mildly treated $\left(\mathrm{HF}_{\mathrm{Na}} \mathrm{s}\right)$ in an aqueous solution of $\mathrm{NaOH}\left(0.2 \%\right.$ wt./v) at $20^{\circ} \mathrm{C}$ for $48 \mathrm{~h}$, following a procedure analogous to that reported in the literature [18].

Except where indicated otherwise, chemicals were purchased from Sigma-Aldrich and used as received.

\subsection{Preparation of the $M C O S$}

MCOs were obtained from PBAT by CDP at high dilution (1\% wt./v), at solvent reflux temperature and in the presence of $3 \mathrm{~mol} \%$ of a transesterification catalyst (namely $n$-dibutyltin(IV) oxide, $n$ $\mathrm{Bu}_{2} \mathrm{SnO}$, or titanium(IV) 2-ethylhexyloxide, $\left.\mathrm{Ti}(\mathrm{OR})_{4}\right)$, by adapting a procedure already described for other polyesters [3,7]. $o$-Dichlorobenzene (DCB) or $\mathrm{CHCl}_{3}$ were used as the solvent. The work up adopted for CDPs in DCB involved the solvent removal by rotoevaporation at $70^{\circ} \mathrm{C}$ and $2-3$ $\mathrm{mmHg}$ followed by drying in a vacuum oven to constant weight. In the case of $\mathrm{CHCl}_{3}$, the $\mathrm{CDP}$ products were recovered by solvent evaporation at room temperature in a fume hood, followed by careful drying under mild conditions. The CDP yield was determined as weight percentage with respect to the initial amount of PBAT. Reaction times were varied from 3 to 5 days.

The MCOs obtained from the CDP carried out under optimized conditions were used as such (that is non-purified: these are labelled npMCOs in the following where appropriate) or were purified by dissolution in the minimum amount of $\mathrm{CHCl}_{3}$ and elution with $\mathrm{CH}_{2} \mathrm{Cl}_{2}$ /acetone 94/6 v/v through a column of activated basic $\mathrm{Al}_{2} \mathrm{O}_{3}$ (150 mesh). Effective purification was checked by thin layer 
126 chromatography (TLC), elemental analysis and SEC. Purified MCOs were labelled pMCOs in the 127 following where appropriate.

128 Before use MCOs were stored under vacuum in anhydrous conditions.

\subsection{ED-ROP of MCOs}

131 ED-ROPs were carried out on the MCOs in isothermal conditions in a DSC pan. Samples (about 10 $132 \mathrm{mg})$ were rapidly heated up to the selected temperature. The amount of $n-\mathrm{Bu}_{2} \mathrm{SnO}$ catalyst $(0-3$ $133 \mathrm{~mol} \%$ with respect to the MCOs), reaction time $(8-120 \mathrm{~min})$ and temperature $\left(180-200^{\circ} \mathrm{C}\right)$ were 134 varied. The products obtained from the ED-ROPs were then characterized by SEC and DSC and compared with the commercial PBAT.

\subsection{Modification of hemp fibres with the MCOs}

138 On alkali treated $\mathrm{HF}_{\mathrm{Na}} \mathrm{s}$ two modification procedures were performed as follows: (i) npMCOs were 139 pre-adsorbed onto $\mathrm{HF}_{\mathrm{Na}} \mathrm{S}\left(\mathrm{HF}_{\mathrm{Na}}-\mathrm{npMCOs}\right)$ by immersing the fibres in a $\mathrm{CHCl}_{3}$ solution (5\% wt./v) 140 of npMCOs; or (ii) $n$ - $\mathrm{Bu}_{2} \mathrm{SnO}$ was previously adsorbed onto $\mathrm{HF}_{\mathrm{Na}} \mathrm{s}$ through immersion of the fibres 141 in a $\mathrm{CHCl}_{3}$ solution $\left(0.18 \%\right.$ wt./v) of the catalyst and, after $\mathrm{CHCl}_{3}$ evaporation, pMCOs were added 142 as described for (i), obtaining fibres labelled $\mathrm{HF}_{\mathrm{Na}}-\mathrm{C}-\mathrm{pMCOs}$.

143 After $\mathrm{CHCl}_{3}$ evaporation, the MCOs pre-adsorbed on the fibres underwent ED-ROP by simple 144 heating in a P200E semi-automatic hot-plate press (Collin $\mathrm{GmbH}$ ). For $\mathrm{HF}_{\mathrm{Na}}-\mathrm{npMCOs}$ ED-ROPs 145 were carried out at 190 or $200^{\circ} \mathrm{C}$ for 30 or $120 \mathrm{~min}$, while $190^{\circ} \mathrm{C}$ and $30 \mathrm{~min}$ were the conditions 146 adopted for $\mathrm{HF}_{\mathrm{Na}}-\mathrm{C}-\mathrm{pMCOs}$. The so obtained fibres were then washed several times with $\mathrm{CHCl}_{3}$ to remove ungrafted oligoester chains until TLC of the extracts did not reveal any trace of unreacted or ungrafted products. Extracts were analyzed by Fourier transform infrared spectroscopy (FTIR) and

150 A higher amount of $\mathrm{HF}_{\mathrm{Na}}-\mathrm{npMCO}$ fibres was obtained by fixing uncut, combed and slightly 151 stretched $\mathrm{HF}_{\mathrm{Na}}$ fibres to a frame and immersing them in a $\mathrm{CHCl}_{3}$ solution $(2.5 \% \mathrm{wt} . / \mathrm{v}$ ) of npMCOs 152 (30 wt.\% MCOs vs. fibres). After solvent evaporation at room temperature and pressure, ED-ROP 153 of the pre-adsorbed npMCOs was performed at $190^{\circ} \mathrm{C}$ for $30 \mathrm{~min}$. The ensuing $\mathrm{HF}_{\mathrm{Na}}-\mathrm{npMCO}$ fibres 154 were carefully washed with $\mathrm{CHCl}_{3}$ and dried at $60^{\circ} \mathrm{C}$ in a ventilated oven.

\subsection{Preparation of hemp fibre/PBAT composites}

157 Preliminary PBAT-based composites containing low amounts (less than 5 wt.\%) of $\mathrm{HF}, \mathrm{HF}_{\mathrm{Na}}$, 158 $\mathrm{HF}_{\mathrm{Na}}-\mathrm{C}-\mathrm{pMCO}$, or $\mathrm{HF}_{\mathrm{Na}}-\mathrm{npMCO}$ fibres were prepared by placing slightly stretched fibres between 
two $0.3 \mathrm{~mm}$ thick sheets of PBAT and by moulding the resulting "sandwich" in the semi-automatic press at $180^{\circ} \mathrm{C}$, i.e. above $T_{m}$, for $1 \mathrm{~min}$ at 5 bar and then 4 min at $160 \mathrm{bar}$.

PBAT-based composites (10x10x0.1 cm sheets) containing higher amounts (about $40 \mathrm{wt} \%$ ) of uncut oriented $\mathrm{HF}, \mathrm{HF}_{\mathrm{Na}}$, and $\mathrm{HF}_{\mathrm{Na}}-\mathrm{npMCO}$ fibres were then prepared by compression moulding through a two-step method: (i) impregnation of combed and slightly stretched fibres into melted PBAT $\left(180^{\circ} \mathrm{C}, 8 \mathrm{~min}\right)$ and achievement of sheets by moulding at $180^{\circ} \mathrm{C}, 1 \mathrm{MPa}, 7 \mathrm{~min}$; (ii) moulding $\left(150^{\circ} \mathrm{C}, 1 \mathrm{MPa}, 3 \mathrm{~min}\right)$ two so-obtained sheets with fibres oriented in the same direction.

\subsection{Characterizations}

SEC analyses were carried out using a Perkin Elmer chromatography system with a Diode Array Detector $235 \mathrm{C}$ and operating at a flow rate of $1 \mathrm{~mL} / \mathrm{min}$. $\mathrm{CHCl}_{3}$ was used both as solvent and eluent. Column set: $10^{3}$ (PL GEL, $30 \mathrm{~cm}, 5 \mu \mathrm{m}$ ), $500 \AA$ (PL GEL, $30 \mathrm{~cm}, 5 \mu \mathrm{m}$ ), $500 \AA$ (PL GEL, $30 \mathrm{~cm}, 10 \mu \mathrm{m}), 100 \AA$ (Styragel HR1, $30 \mathrm{~cm}, 5 \mu \mathrm{m})$. PBAT pellets were analysed with the following column set: $10^{5}$ (Styragel HR5, $30 \mathrm{~cm}, 5 \mu \mathrm{m}$ ), 10 $10^{4}, 10^{3}, 500 \AA$ (PL GEL, $30 \mathrm{~cm}, 5 \mu \mathrm{m}$ ). TLC analysis was carried out by using $\mathrm{CH}_{2} \mathrm{Cl}_{2} /$ acetone $94 / 6 \mathrm{v} / \mathrm{v}$ as eluent.

Elemental analysis was carried out by inductively coupled plasma-optical emission spectroscopy (ICP-OES) using a Fisons Instruments Horizon instrument to evaluate Sn content in selected MCOs samples.

Room temperature ${ }^{1} \mathrm{H}$ NMR spectrum of PBAT dissolved in deuterochloroform $\left(\mathrm{CDCl}_{3}\right)$ was obtained with a Bruker Avance-500 spectrometer (no internal chemical shift reference).

FTIR spectroscopic characterization of PBAT, MCOs, fibres, and extracts from the fibre washing was performed with a Perkin Elmer Spectrum Two ${ }^{\text {TM }}$ FTIR spectrometer operating in the attenuated total reflectance (ATR) mode and recording absorbance spectra in the wave number range 4000-400 $\mathrm{cm}^{-1}$.

Room temperature wide angle X-ray diffraction (WAXD) spectra of hemp fibres were obtained using a Siemens diffractometer model D-500 equipped with a Siemens FK 60-10 2000W tube (Cu $\mathrm{K}_{\alpha}$ radiation, $\lambda=0.154 \mathrm{~nm}$ ). The operating voltage and current were $40 \mathrm{kV}$ and $40 \mathrm{~mA}$, respectively. Data were collected from 5 to $40^{\circ} 2 \theta$ at $0.02^{\circ} 2 \theta$ intervals. The percentage crystallinity index $(C I)$ of cellulose in hemp fibres was determined by extracting the three crystalline peaks between 10 and $30^{\circ} 2 \theta$ from the diffraction intensity profiles with a non-linear curve-fitting process [19,20]. A peak fitting commercial software (OriginPro 2015, OriginLab Co.) was used, assuming for each peak Lorentzian functions. The $C I$ was calculated from the ratio of the area of the crystalline peaks to the total area. 
192 A Mettler DSC $821^{\mathrm{e}}$ calorimeter was used for thermal characterization of the materials and for 193 performing small-scale ED-ROPs in the DSC pan. For characterization of the products obtained 194 from CDPs a single heating run between 0 and $200^{\circ} \mathrm{C}$ (scan rate $20^{\circ} \mathrm{C} / \mathrm{min}$ ) was performed. 195 Heating-cooling-heating cycles from 0 up to $200^{\circ} \mathrm{C}$ (scan rate $20^{\circ} \mathrm{C} / \mathrm{min}$ ) were applied for PBAT 196 pellets and products obtained from ED-ROPs.

197 Thermogravimetric analysis (TGA) measurements were performed with a PerkinElmer TGA7 198 analyzer operating in dynamic mode. Samples of PBAT and fibres of about $10 \mathrm{mg}$ were heated at $19920{ }^{\circ} \mathrm{C} / \mathrm{min}$ under $\mathrm{O}_{2}$ from 50 to $900^{\circ} \mathrm{C}$ (gas flow rate $40 \mathrm{~mL} / \mathrm{min}$ ). The water content (mass loss at $\left.200150^{\circ} \mathrm{C}\right)$, the decomposition temperatures corresponding to $5 \mathrm{wt} . \%$ mass loss $\left(\mathrm{T}_{5}\right)$ and the maximum 201 rate of decomposition $\left(T_{\operatorname{Vmax}}\right)$ were determined. Samples of composites of about $10 \mathrm{mg}$ were heated 202 at $10{ }^{\circ} \mathrm{C} / \mathrm{min}$ under $\mathrm{N}_{2}$ from 30 to $750^{\circ} \mathrm{C}$ (gas flow rate $40 \mathrm{~mL} / \mathrm{min}$ ) for the evaluation of the fibre 203 content. The onset $\left(T_{\text {onset }}\right)$ and the $T_{V \max }$ degradation temperatures were determined. For reference 204 purpose, neat PBAT and all HFs were analysed in the same experimental conditions.

205 Morphological analysis was carried out by scanning electron microscopy (SEM) using a Hitachi 206 TM3000 benchtop SEM microscope operating at $15 \mathrm{kV}$ acceleration voltage. Fibres and fragile 207 surfaces of composites fractured in liquid $\mathrm{N}_{2}$ were sputtered with gold using a Quorum Q150R ES 208 209 sputter coater (2M Strumenti) at $20 \mathrm{~mA}$ for 5 min prior to the observation. Moreover, a FE-SEM Zeiss Supra 40VP was used for a deeper morphological analysis of the samples after dynamic-

211 Uniaxial tensile tests were performed by using a displacement-controlled dynamometer (Instron $2125565)$ according to the ASTM D412 TC standard method $\left(25^{\circ} \mathrm{C}\right.$, load cell $5 \mathrm{kN}$, constant crosshead 213 speed $\left.20 \mathrm{~mm} \mathrm{~min}^{-1}\right)$ on rectangular specimens $(80 \times 10 \times 1 \mathrm{~mm})$. Tensile modulus $(E)$, stress $\left(\sigma_{\mathrm{b}}\right)$ and 214 strain $\left(\varepsilon_{\mathrm{b}}\right)$ at break were evaluated for neat PBAT matrix and its composites with hemp fibres. 215 Mechanical parameters were measured on more than 6 specimens and the average values were 216 calculated.

217 Dynamic-mechanical measurements were carried out on rectangular specimens (40x10x1 mm) with 218 a strain-controlled rotational rheometer MCR301 (Anton Paar). The range of viscoelastic linearity 219 was determined through amplitude sweep tests performed at $1 \mathrm{~Hz}$ and $25^{\circ} \mathrm{C}$ in the strain range $220 \quad 0.01 \div 100 \%$; room temperature frequency sweep tests were then carried out in the torsion mode at $2210.1 \%$ amplitude deformation in the frequency range $0.1 \div 50 \mathrm{~Hz}$, both in longitudinal and transversal 222 direction (i.e., with fibres parallel or perpendicular to the clamp axis, respectively). Elastic (G') and 223 dissipative (G") components of the modulus were determined. 


\section{Results and Discussion}

\subsection{CDP of PBAT}

CDPs of PBAT were carried out by using different solvents ( $\mathrm{DCB}$ or $\left.\mathrm{CHCl}_{3}\right)$, catalysts $\left(\mathrm{Ti}(\mathrm{OR})_{4}\right.$, with $\mathrm{R}=2$-ethylhexyl, or $n$ - $\mathrm{Bu}_{2} \mathrm{SnO}$ ) and/or reaction times (3-5 days), as described in the Materials and methods section and summarized in Table 1.

Table 1. CDPs of PBAT carried out at high dilution (1 \%wt./v) and SEC characterization.

\begin{tabular}{|c|c|c|c|c|c|}
\hline Run & Solvent & Catalyst & $\begin{array}{l}\text { Reaction time } \\
\text { (days) }\end{array}$ & $\begin{array}{c}\text { Product Yield }^{\mathrm{a}} \\
(\text { wt.\%) }\end{array}$ & $\begin{array}{c}\text { Fraction with } M p<4000^{a} \\
(\%)\end{array}$ \\
\hline 1 & DCB & $\mathrm{Ti}(\mathrm{OR})_{4}^{\mathrm{b}}$ & 3 & 88 & 16 \\
\hline 2 & DCB & $\mathrm{Ti}(\mathrm{OR})_{4}{ }^{\mathrm{b}}$ & 4 & 71 & 33 \\
\hline 3 & $\mathrm{DCB}$ & $n-\mathrm{Bu}_{2} \mathrm{SnO}$ & 3 & 25 & 85 \\
\hline 4 & $\mathrm{CHCl}_{3}$ & $n-\mathrm{Bu}_{2} \mathrm{SnO}$ & 5 & 100 & 100 \\
\hline 5 & $\mathrm{CHCl}_{3}$ & $n-\mathrm{Bu}_{2} \mathrm{SnO}$ & 4 & 100 & 100 \\
\hline 6 & $\mathrm{CHCl}_{3}$ & $n-\mathrm{Bu}_{2} \mathrm{SnO}$ & 3 & 100 & 100 \\
\hline
\end{tabular}

$234{ }^{a}$ Calculated as weight percentage of recovered reaction products with respect to the initial amount 235 of PBAT.

$236{ }^{\mathrm{b}}$ Obtained from SEC analysis.

$237{ }^{\mathrm{c}}$ R: 2-ethylhexyl.

239 The reaction was conveniently monitored by SEC analysis. SEC traces of the starting PBAT pellets 240 and of the equilibrated CDP mixtures obtained under selected experimental conditions are 241 compared in Fig. 1. Depending on reaction conditions, the SEC traces present either a major peak of 242 relatively high molecular weight (Run 1 and 2) or a series of peaks of low molecular weight (Run 243 6). In the former case, CDP of PBAT does not occur to an appreciable extent and PBAT is 244 practically recovered unaltered; in the latter reactions the MCOs are successfully formed, as 245 evidenced by the series of peaks corresponding to MCOs of different ring sizes and, possibly, 246 compositions (since PBAT is a copolyester with aliphatic-aliphatic and aliphatic-aromatic ester 247 linkages having non-identical chemical reactivity). The relative amount of each species present in 248 the reaction product was calculated from the SEC traces as percentage area. 


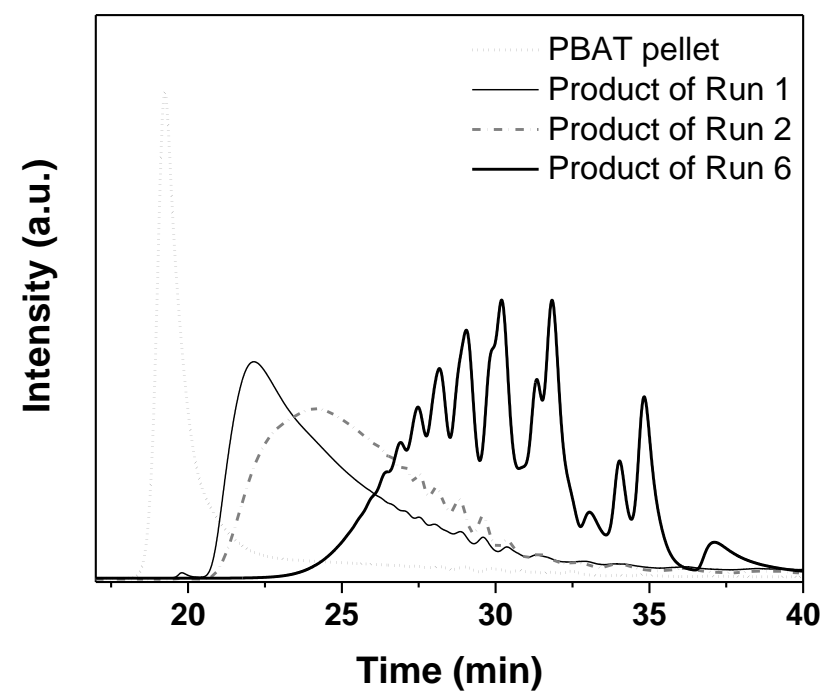

Fig. 1. SEC traces of PBAT and some selected products obtained from its CDP. (1-column fitting 252 image)

254 No significant amounts of low molecular weight species were obtained with the Ti-based catalyst 255 (Run 1 and 2), but MCOs were successfully prepared by CDP in both solvents when $n-\mathrm{Bu}_{2} \mathrm{SnO}$ was 256 the catalyst. However, when DCB was used as the solvent (Run 3) the work-up following the 257 reaction led to the recovery of a very low amount of MCOs product (25\%). This is ascribable to the 258 loss of lower molecular weight fraction of MCOs, considering that the monomeric cyclic $n$-butyl 259 adipate is expected to be the most volatile one.

260 By using $\mathrm{CHCl}_{3}$ as solvent, which has a much lower boiling point than DCB, the work-up was 261 easier, as indicated in the Materials and methods section, and quantitative yields in MCOs were 262 obtained (Run 4-6). The effect of the reaction time was also evaluated carrying out the CDP with 263 the $\mathrm{CHCl}_{3} / n-\mathrm{Bu}_{2} \mathrm{SnO}$ pair: as evidenced in Table 1 and in Fig. 2, 3 days at reflux temperature are 264 necessary for obtaining the MCOs in high yield (Run 6). 


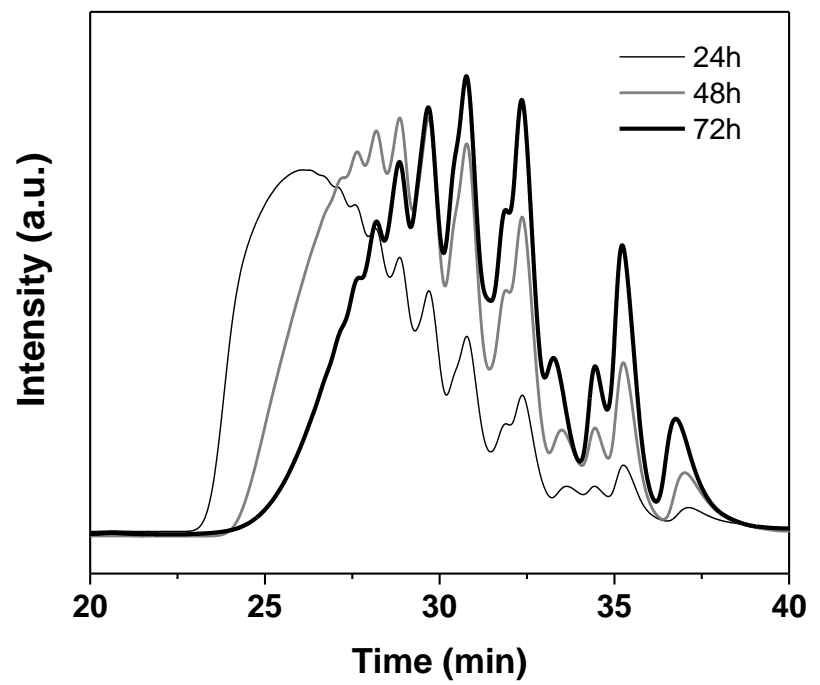

268 Fig. 2. SEC traces of products obtained from CDP of PBAT carried out at different reaction times 269 (see Table 1, Runs 4-6). (1-column fitting image)

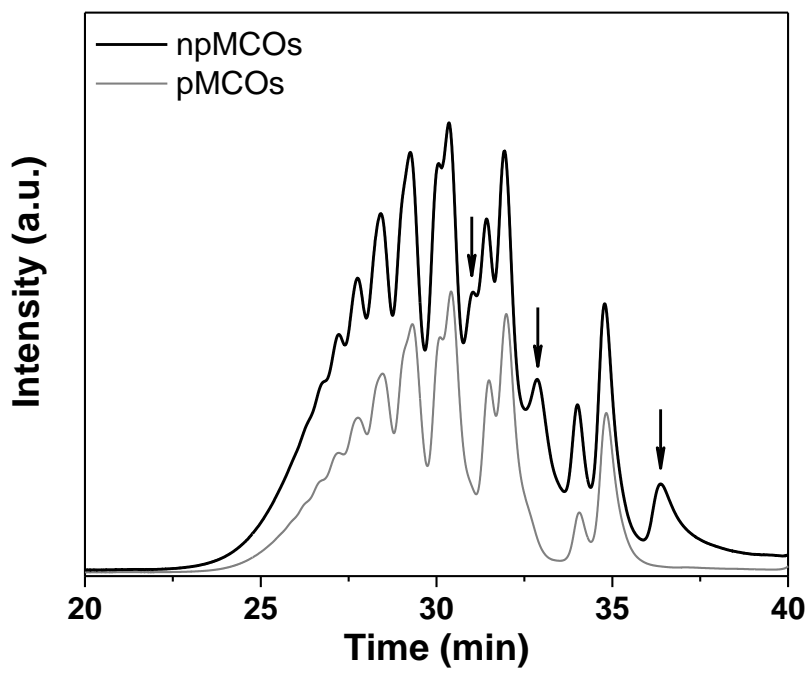

272 Fig. 3. SEC traces of the MCOs obtained as in Run 6 of Table 1, before and after purification. (1273 column fitting image)

Accordingly, the MCOs obtained from Run 6 were selected to perform successive ED-ROP experiments and HF modification. The MCOs were used either as directly obtained from the CDP (npMCOs), or were purified by adsorptive filtration on $\mathrm{Al}_{2} \mathrm{O}_{3}$ (pMCOs) [7] to remove as much as possible any residue of transesterification catalyst and possible traces of linear oligomeric species. A yield of 65 wt.\% was found after filtration. TLC carried out on the MCOs before and after purification indicated the removal of the most polar species. Furthermore, in the SEC traces of 281 purified MCOs the peaks at 240, 480 and $750 \mathrm{~g} / \mathrm{mol}$, probably ascribable to linear oligomeric species [21], disappeared (Fig. 3 and Table 2). The presence of the tin-based catalyst used for the 
CDP was checked by elemental analysis for Sn both in npMCOs and pMCOs. A content of 1.44 wt.\% of Sn (that corresponds to the $3 \mathrm{~mol} \%$ catalyst introduced) was found in npMCOs, which were isolated without any washing after CDP; whereas after the filtration on $\mathrm{Al}_{2} \mathrm{O}_{3}$ practically all the catalyst was removed from pMCOs, since only traces $(<0.1 \%)$ of tin were detected. DSC analysis showed a similar thermal behaviour for the MCOs both before and after purification. Thus, a broad endothermic peak centred at about $50^{\circ} \mathrm{C}$ is observed upon heating (this low melting range supports the considerations on the relative volatility of the MCOs previously done).

291 Table 2. SEC Characterization of the MCOs before and after purification.

\begin{tabular}{c|c|c|c}
\multicolumn{2}{c|}{ npMCOs } & \multicolumn{2}{c}{ pMCOs } \\
\hline $\begin{array}{c}\boldsymbol{M}_{\mathbf{p}}^{\mathbf{a}} \\
(\mathbf{g} / \mathbf{m o l})\end{array}$ & $\begin{array}{c}\text { Area } \\
(\boldsymbol{\%})\end{array}$ & $\begin{array}{c}\boldsymbol{M}_{\mathbf{p}}^{\mathbf{a}} \\
(\mathbf{g} / \mathbf{m o l})\end{array}$ & $\begin{array}{c}\text { Area } \\
(\boldsymbol{\%})\end{array}$ \\
\hline 3930 & 9.7 & 3950 & 9.4 \\
2420 & 4.8 & 2410 & 4.3 \\
2020 & 6.5 & 2000 & 7.8 \\
1630 & 9.3 & 1600 & 11.1 \\
1250 & 12.4 & 1230 & 15.2 \\
990 & 6.3 & 980 & 6.7 \\
910 & 9.0 & 900 & 14.1 \\
750 & 3.9 & - & - \\
680 & 6.5 & 680 & 6.8 \\
600 & 10.2 & 600 & 14.3 \\
480 & 6.1 & - & - \\
370 & 3.5 & 370 & 2.3 \\
320 & 7.0 & 320 & 8.1 \\
240 & 4.8 & - & -
\end{tabular}

${ }^{\text {a }}$ Molecular weight at peak.

\subsection{ED-ROP of the MCOs}

295 Several ED-ROP runs on the MCOs were carried out under $\mathrm{N}_{2}$ in a pan placed in the DSC furnace with the aim of investigating the influence of $n-\mathrm{Bu}_{2} \mathrm{SnO}$ concentration, reaction time and temperature on molecular and thermal characteristics of the ensuing polyesters. For this purpose, samples of the MCOs containing different amounts of $n-\mathrm{Bu}_{2} \mathrm{SnO}$ catalyst were obtained by mixing solutions (using $\mathrm{CHCl}_{3}$ as a solvent) of npMCOs, which were assumed to contain unvaried (with respect to the amount introduced for the $\mathrm{CDP}$ ) $n-\mathrm{Bu}_{2} \mathrm{SnO}$ content ( $3 \mathrm{~mol} \%$ ), and of pMCOs (assumed to be completely free of catalyst). As shown by SEC traces of Fig. 4, the presence of $n$ - 
$302 \mathrm{Bu}_{2} \mathrm{SnO}$ is necessary for efficient ED-ROPs of MCOs in the selected conditions. Products with 303 higher molecular weights were obtained by increasing the amount of $n-\mathrm{Bu}_{2} \mathrm{SnO}$.
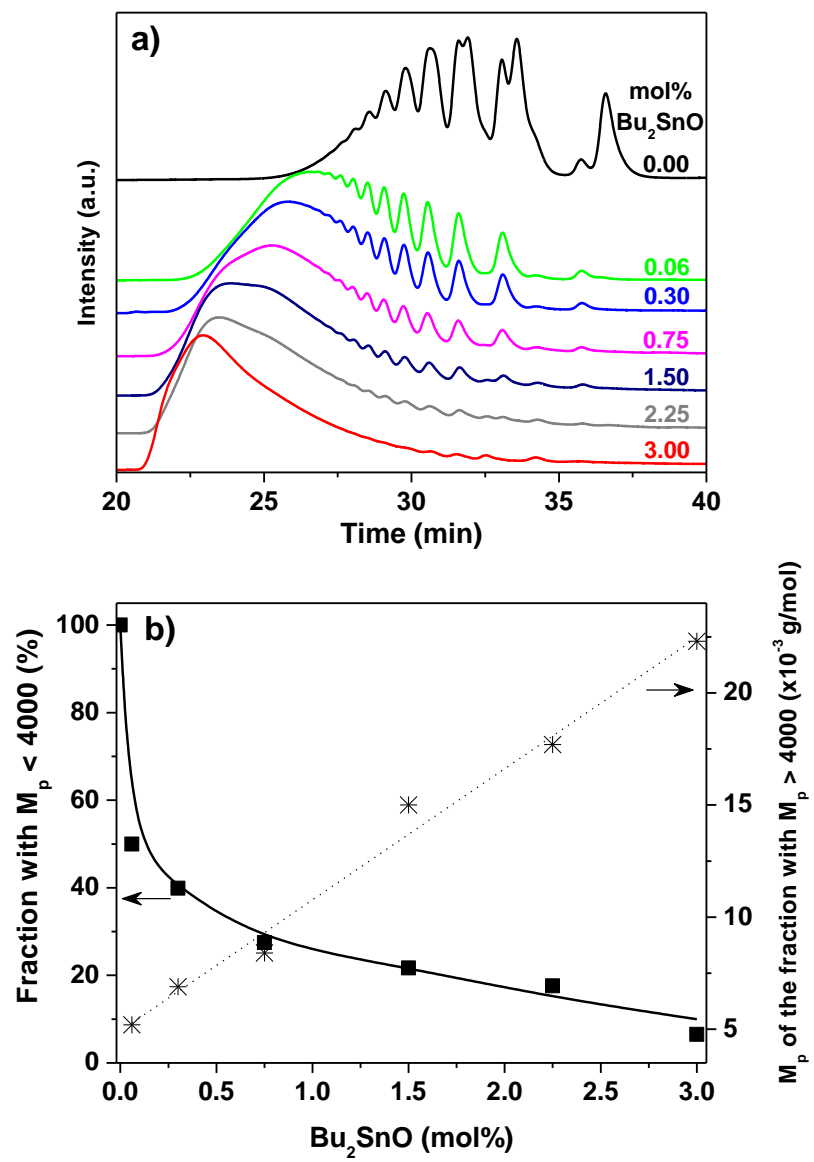

305 Fig. 4. SEC traces of products obtained from ED-ROPs of the MCOs $\left(180^{\circ} \mathrm{C}, 120 \mathrm{~min}\right)$ carried out 306 by varying the amount of $n-\mathrm{Bu}_{2} \mathrm{SnO}$ (a); Plots of $M_{p}$ and Fraction with $M_{p}<4000 v$ s. $n-\mathrm{Bu}_{2} \mathrm{SnO}$ amount (b). (1-column fitting image)

309 Thus ED-ROP runs were carried out on npMCOs (that is containing 3 mol\% $n$ - $\mathrm{Bu}_{2} \mathrm{SnO}$ ) varying the 310 reaction time. Data obtained from SEC analysis of ED-ROP products shown in Fig. 5 indicate that 311 ED-ROP of npMCOs takes place to a certain extent already after $8 \mathrm{~min}$ and the molecular weight of 312 ED-ROP products being formed increased rapidly in the first $30 \mathrm{~min}$ in the DSC pan. Reaction 313 times longer than $30 \mathrm{~min}$ did not lead to substantial further increase of the molecular weight of the 314 products. 

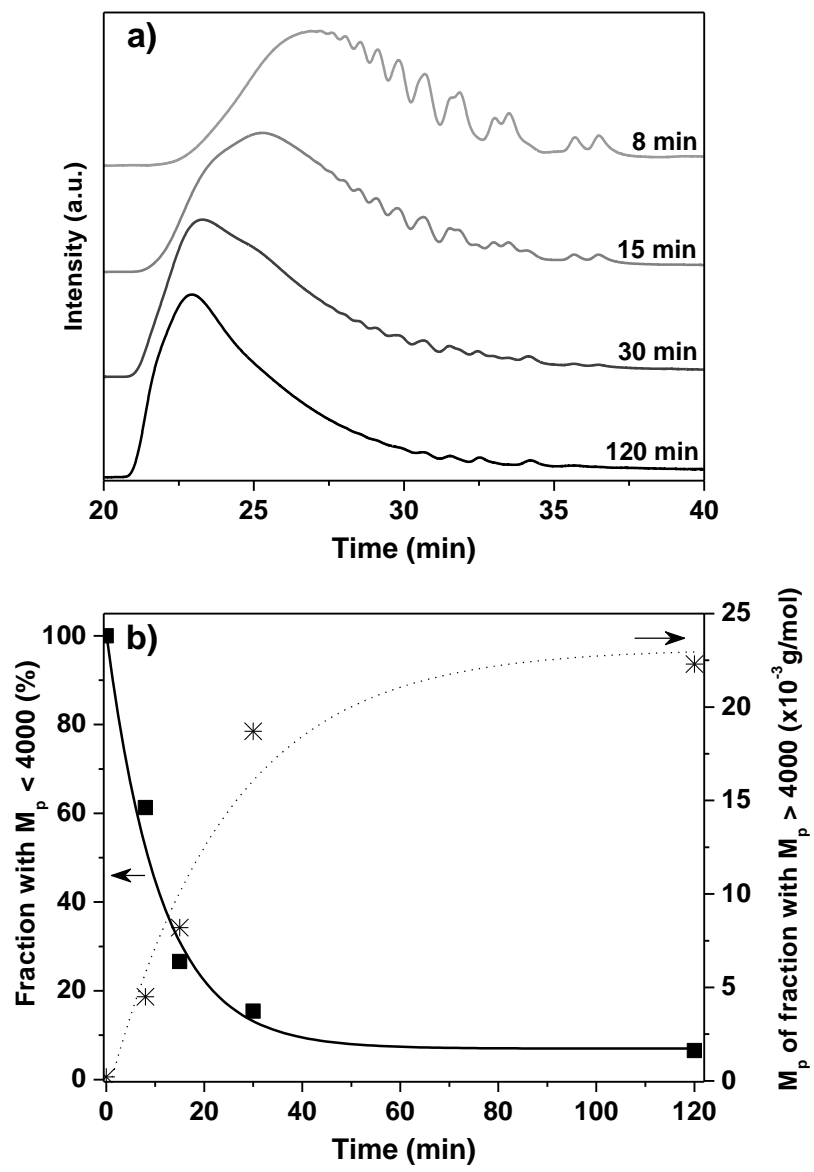

Fig. 5. SEC traces of products obtained from ED-ROPs of the MCOs $\left(3 \mathrm{~mol} \% n-\mathrm{Bu}_{2} \mathrm{SnO}, 180^{\circ} \mathrm{C}\right)$ carried out by varying the reaction time (a); Plots of $M_{p}$ and Fraction with $M_{p}<4000 v s$. reaction time (b). (1-column fitting image)

ED-ROP runs were finally carried out on npMCOs fixing reaction time at $30 \mathrm{~min}$ and varying the temperature $\left(180,190,200^{\circ} \mathrm{C}\right)$. Obtained SEC data (not shown here) indicate that for temperatures higher than $190^{\circ} \mathrm{C}$ the molecular weight reaches a plateau value of about $21000 \mathrm{~g} / \mathrm{mol}$ and the amount of low molecular species is lower than $10 \%$.

DSC analysis of the ED-ROP products (data not shown) confirmed the results obtained from SEC analysis. The product obtained from ED-ROP of npMCOs carried out at $190^{\circ} \mathrm{C}$ for 30 min presents a thermal behaviour similar to that of the original PBAT pellets (melting temperature about $120^{\circ} \mathrm{C}$ ).

\subsection{Surface modifications of hemp fibres}

329 Raw hemp fibres, previously freed of shives as indicated in the Materials and methods section, were considered for the preparation of eco-composites based on Ecoflex ${ }^{\circledR}$, a commercial grade of the biodegradable PBAT copolyester. In order to improve their compatibility with the polymer matrix, the fibres were firstly treated under mild conditions with an aqueous solution of $\mathrm{NaOH}$ to make 
333 their functional groups more accessible for successive reactions. Natural cellulosic fibres are 334 complex assemblies including cellulose, hemicellulose, lignin, pectin, waxes and water-soluble 335 substances [22]. The removal of the non-cellulosic materials from the fibre surface is beneficial for 336 interaction with polymer matrices and propaedeutic for the successive chemical modification. 337 Among the proposed methods, alkali treatment is widely used for removing non-cellulosic 338 components and part of the amorphous cellulose [23,24]. However, this treatment can significantly 339 modify the crystalline structure of the fibres. The mild conditions adopted in this work for treating 340 HFs [18], resulted in better separated fibres with cleaner surface topography (Fig. 6 a,b) due to a 341 partial removal of non-cellulosic components.
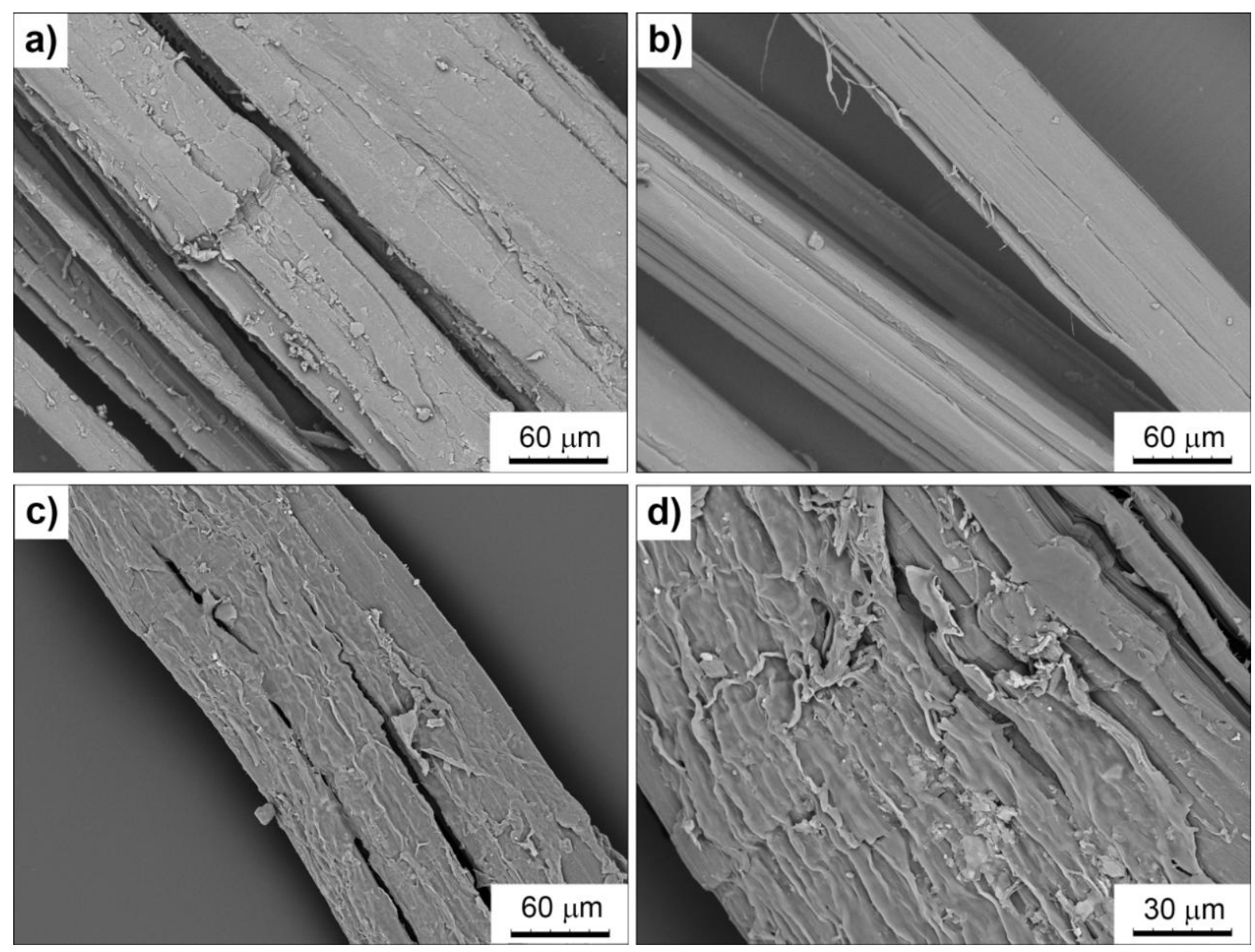

Fig. 6. SEM micrographs of: (a) $\mathrm{HFs}$, (b) $\mathrm{HF}_{\mathrm{Na}} \mathrm{s}$, and (c,d) $\mathrm{HF}_{\mathrm{Na}}$-npMCOs. (2-column fitting image)

ATR-FTIR analysis of $\mathrm{HF}$ and $\mathrm{HF}_{\mathrm{Na}}$ fibres confirmed SEM observations indicating the removal of

346 hemicellulose component as a consequence of the $\mathrm{NaOH}$ treatment. In particular, as shown in Fig.

347 , the intensity of the peak at $1735 \mathrm{~cm}^{-1}$, characteristic of the $\mathrm{C}=\mathrm{O}$ stretching vibration of carboxyl 348 and acetyl moieties in hemicelluloses (xyloglucan) [25], decreases as well as that of the peak at $3491243 \mathrm{~cm}^{-1}$, which corresponds to the C-O linkage, as found in guaiacyl aromatic methoxyl and 350 acetyl moieties of xyloglucan [25]. On the other hand, the peaks at 2920 and $2850 \mathrm{~cm}^{-1}$ 351 corresponding to the $\mathrm{C}-\mathrm{H}$ stretching of lignin and cellulose [26,27] become more evident. 


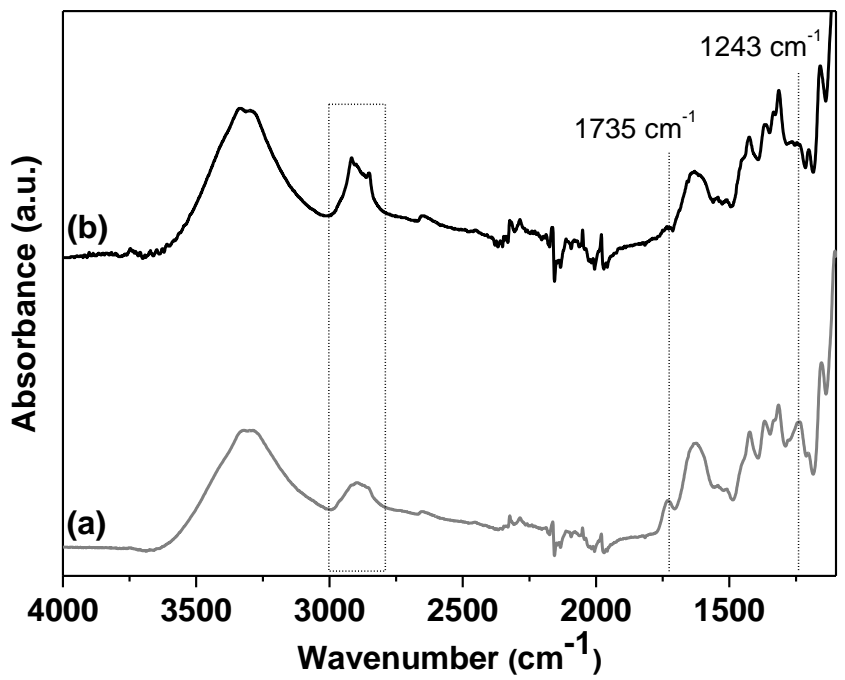

353 Fig. 7. ATR-FTIR spectra of: (a) $\mathrm{HF}$, and (b) $\mathrm{HF}_{\mathrm{Na}}$ fibres. (1-column fitting image)

355 No great differences in the crystalline structure as well as in the crystallinity index $(C I)$ were found 356 before and after the mild alkali treatment of hemp fibres used here. Indeed, both diffractograms 357 (Fig. 8) show the four XRD reflections typical of cellulose I at 15, 16.4, 22.6, and $34.3^{\circ} 2 \theta$ 358 corresponding, respectively, to (101), (10-1), (002), and (040) crystallographic planes [28,29].

359 By peak deconvolution a $C I$ value of $85 \%$ was calculated for $\mathrm{HFs}$ and of $87 \%$ for $\mathrm{HF}_{\mathrm{Na}}$, that is a 360 slightly higher value, also considering the experimental error intrinsic to the technique. However, it 361 is worth mentioning that the fibre crystallinity index is generally used for comparison purpose 362 before and after different treatments rather than to evaluate absolute crystallinity [30-32]. Moreover, 363 the two peaks at 15 and $16.5^{\circ}$ appeared partially overlapped, indicating that the $\mathrm{HF}_{\mathrm{Na}}$ fibres still 364 contain a certain amount of amorphous material [33], as expected following a mild alkali treatment 365 as in the present case; when these two peaks are well separated and pronounced the fibres contain 366 higher amounts of crystalline cellulose. 

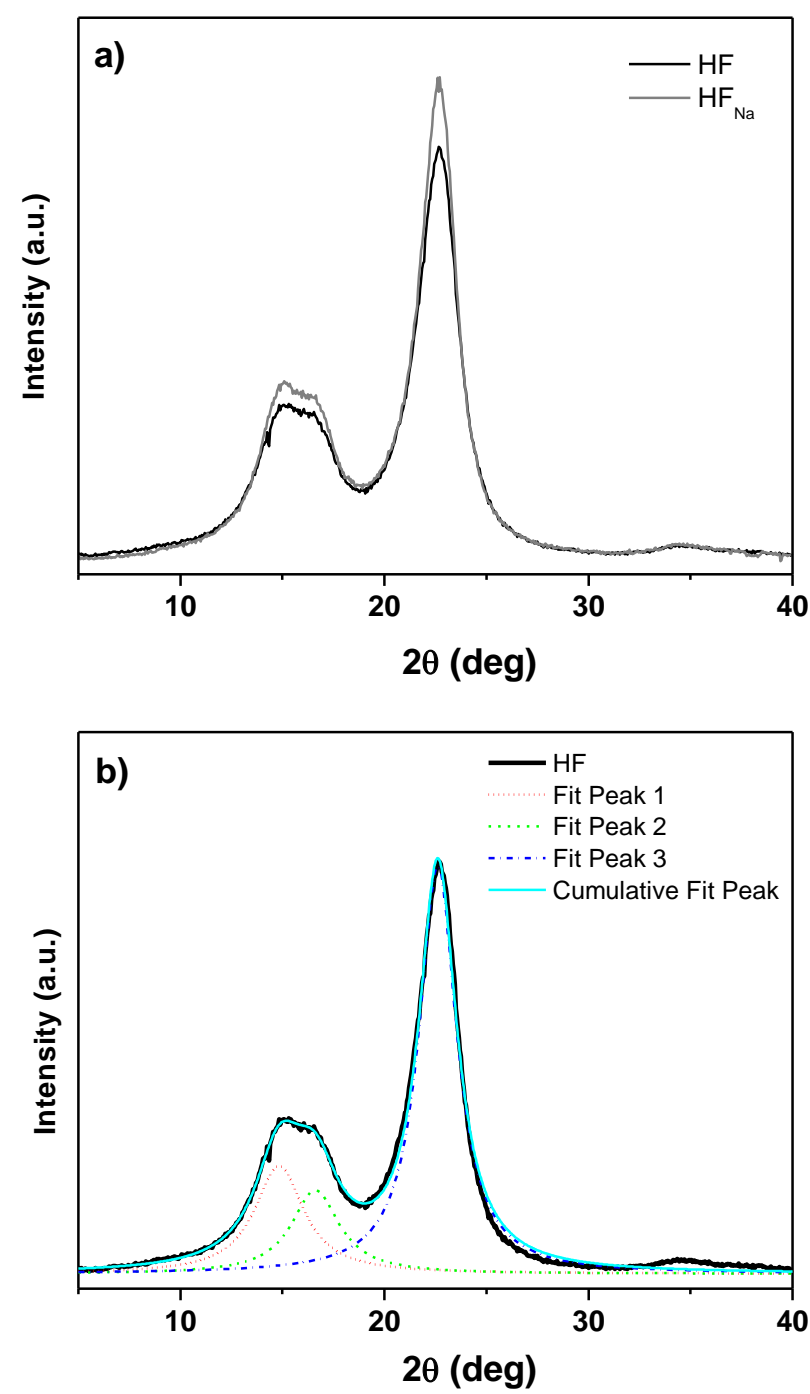

Fig. 8. XRD diffractograms of: (a) $\mathrm{HF}$ and $\mathrm{HF}_{\mathrm{Na}}$ fibres, and (b) example of peak deconvolution of the XRD pattern of HFs. (1-column fitting image)

Thermal and thermo-oxidative stability of hemp fibres before and after the treatment with sodium hydroxide and the subsequent surface modification with the MCOs (see later in the text) were investigated by TGA analysis in dynamic mode. Relevant TGA data collected are summarized in

375 Table 3. The fibre treatment with $\mathrm{NaOH}$ slightly increases the temperature at which the fibres start 376 to degrade under $\mathrm{O}_{2}$ atmosphere; indeed, $T_{5}$ values of $\mathrm{HF}_{\mathrm{Na}} \mathrm{S}$ is about 10 higher than that of HFs. 377 This can be explained by the partial removal of non-cellulosic and amorphous cellulose components 378 (which decompose at lower temperatures) with consequent slight increase in crystallinity. Both 379 under $\mathrm{O}_{2}$ and $\mathrm{N}_{2}$ atmosphere, the $T_{\text {Vmax }}$ values before and after $\mathrm{NaOH}$ treatment are quite similar. 
381 Table 3. Water content at $150^{\circ} \mathrm{C}$ and $T_{5}$ and $T_{V \operatorname{Vmax}}$ decomposition temperatures of hemp fibres 382 determined by TGA.

\begin{tabular}{c|c|c|c|c}
\multicolumn{2}{c|}{} & \multicolumn{2}{|c|}{ Under $\boldsymbol{O}_{2}$} & Under $\boldsymbol{N}_{2}$ \\
\hline Sample & $\begin{array}{c}\mathbf{H}_{\mathbf{2}} \mathbf{O} \text { content @ } \mathbf{1 5 0}^{\circ} \mathbf{C} \\
(\mathbf{w t .} \%)\end{array}$ & $\begin{array}{c}\boldsymbol{T}_{5} \\
\left({ }^{\circ} \mathbf{C}\right)\end{array}$ & $\begin{array}{c}\boldsymbol{T}_{\text {Vmax }} \\
\left({ }^{\circ} \mathbf{C}\right)\end{array}$ & $\begin{array}{c}\boldsymbol{T}_{\text {Vmax }} \\
\left({ }^{\circ} \mathbf{C}\right)\end{array}$ \\
\hline HFs & 2.2 & 307 & 358 & 374 \\
$\mathbf{H F}_{\mathbf{N a}} \mathbf{S}$ & 2.5 & 315 & 355 & 371 \\
$\mathbf{H F}_{\mathbf{N a}}-\mathbf{C}-\mathbf{p M C O s}$ & 1.7 & 327 & 368 & - \\
$\mathbf{H F}_{\mathbf{N a}}-\mathbf{n p M C O S}$ & 1.8 & 325 & 369 & 378
\end{tabular}

384 The surface modification of $\mathrm{HF}_{\mathrm{Na}} \mathrm{s}$ with the synthesized MCOs could in principle be accomplished 385 through different procedures:

386 (i) adsorption of the MCOs onto $\mathrm{HF}_{\mathrm{Na}} \mathrm{s}$ followed by ED-ROP directly during mixing with PBAT

(iii) previous anchorage of the transesterification catalyst onto $\mathrm{HF}_{\mathrm{Na}} \mathrm{S}$, subsequent adsorption of purified pMCOs, that is cleaned by catalyst through elution on $\mathrm{Al}_{2} \mathrm{O}_{3}$, and ED-ROP prior to fibres incorporation in the PBAT matrix.

The easiest and fastest strategy (i) unfortunately gave composite materials with no appreciable improvement in their final properties [17]; this could be ascribed to uncontrolled ED-ROP conditions and too short residence times in the mixer. As a consequence, (ii) and (iii) compatibilization strategies appeared more promising and were further investigated.

As detailed in the Materials and methods section, two procedures for modification of $\mathrm{HF}_{\mathrm{Na}} \mathrm{S}$ with the MCOs, respectively starting from npMCOs or pMCOs macrocycles, were followed. The ED-ROP of the MCOs adsorbed onto the hemp fibres was performed by using the reaction conditions $\left(190^{\circ} \mathrm{C}, 30 \mathrm{~min}\right)$ established by the polymerization experiments previously carried out in the DSC pan. The two MCO-modified fibre samples obtained after ED-ROP and extraction of unreacted or ungrafted products were called $\mathrm{HF}_{\mathrm{Na}}-\mathrm{npMCOs}$ and $\mathrm{HF}_{\mathrm{Na}}-\mathrm{C}-\mathrm{pMCOs}$, respectively.

The presence of PBAT oligomers ( $M_{\mathrm{p}}$ ranging from 5000 to $7000 \mathrm{~g} / \mathrm{mol}$ ) in the soluble fractions obtained by washing both MCO-modified fibre samples with $\mathrm{CHCl}_{3}$ was ascertained by FTIR and SEC analyses. This finding confirms that unreacted macrocycles and/or ungrafted linear oligomers were effectively removed from hemp fibres. As a consequence, the reacted portion which remains on the fibres is actually grafted. The ATR-FTIR analysis of fibres modified by both procedures (ii) and (iii) featured the presence on their surface of the PBAT moieties, as desired. In particular, the 
410 peaks at 1715 and $1270 \mathrm{~cm}^{-1}$, respectively due to the stretching modes of $\mathrm{C}=\mathrm{O}$ and $\mathrm{C}-\mathrm{O}$ of the ester 411 linkages belonging to the polyester chains, were observed (Fig. 9). It is worth noticing that these 412 peaks are absent in the spectrum of alkali treated $\mathrm{HF}_{\mathrm{Na}}$, that only exhibits a feeble signal ascribable 413 to carbonyl groups centred at $1735 \mathrm{~cm}^{-1}$.

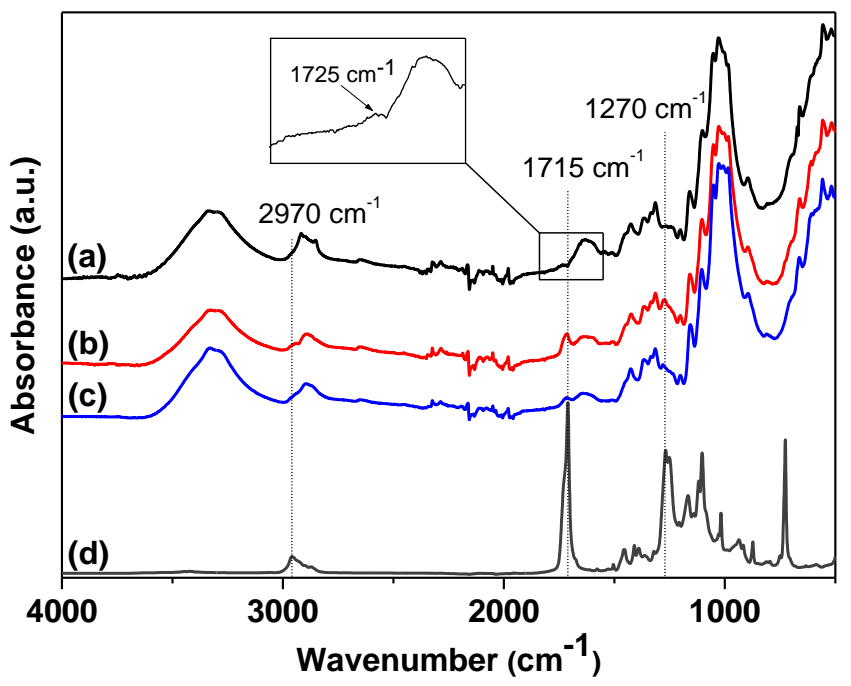

416 Fig. 9. ATR-FTIR spectra of: (a) $\mathrm{HF}_{\mathrm{Na}} \mathrm{s}$, (b) $\mathrm{HF}_{\mathrm{Na}}-n p M C O s$, (c) $\mathrm{HF}_{\mathrm{Na}}-\mathrm{C}-\mathrm{pMCOs}$, and (d) PBAT. 417 Inset shows the ATR-FTIR spectrum of $\mathrm{HF}_{\mathrm{Na}} \mathrm{S}$ in the $1950-1550 \mathrm{~cm}^{-1}$ window. (1-column fitting 418 image)

As shown by the parameters collected in Table 3, the surface modification of $\mathrm{HF}_{\mathrm{Na}} \mathrm{s}$ with the MCOs results in an improvement of the thermo-oxidative stability of the fibres. Moreover, the water content of fibres stored in an ambient environment $\left(\mathrm{T} \approx 25^{\circ} \mathrm{C}, \mathrm{RH} \approx 65 \%\right)$, evaluated as the weight loss at $150^{\circ} \mathrm{C}$, decreases after modification with the MCOs, indicating, as expected, a reduction of the surface hydrophilicity.

425 SEM images of Fig. 6 c,d clearly show the presence of a polymeric layer covering the surface of fibres modified with npMCOs. The increase in thermo-oxidative stability of both samples of MCOmodified fibres can be ascribed to the protection exerted by this polymeric layer, being the thermooxidative decomposition temperatures $T_{5}$ and $T_{V \max }$ recorded for PBAT in the same conditions, respectively 344 and $415^{\circ} \mathrm{C}$.

\subsection{PBAT/hemp fibres eco-composites}

432 In order to evaluate the effect of the fibre modification on the fibre-polymer adhesion, preliminary 433 tests of adhesion were carried out on composites containing < $5 \mathrm{wt} \%$ of $\mathrm{HF}, \mathrm{HF}_{\mathrm{Na}}, \mathrm{HF}_{\mathrm{Na}}-\mathrm{npMCO}$ 434 or $\mathrm{HF}_{\mathrm{Na}}-\mathrm{C}-\mathrm{pMCO}$ fibres prepared by placing slightly stretched fibres between two sheets of PBAT 
(previously obtained by compression moulding of PBAT pellets) and by moulding the resulting "sandwich" in the hot-press, as detailed in the Materials and methods section.

437 The ensuing samples were then fractured in liquid nitrogen and the morphological characterization 438 of their fractured surfaces was performed by SEM. Some representative images taken at the same 439 magnification were shown in Fig. 10.
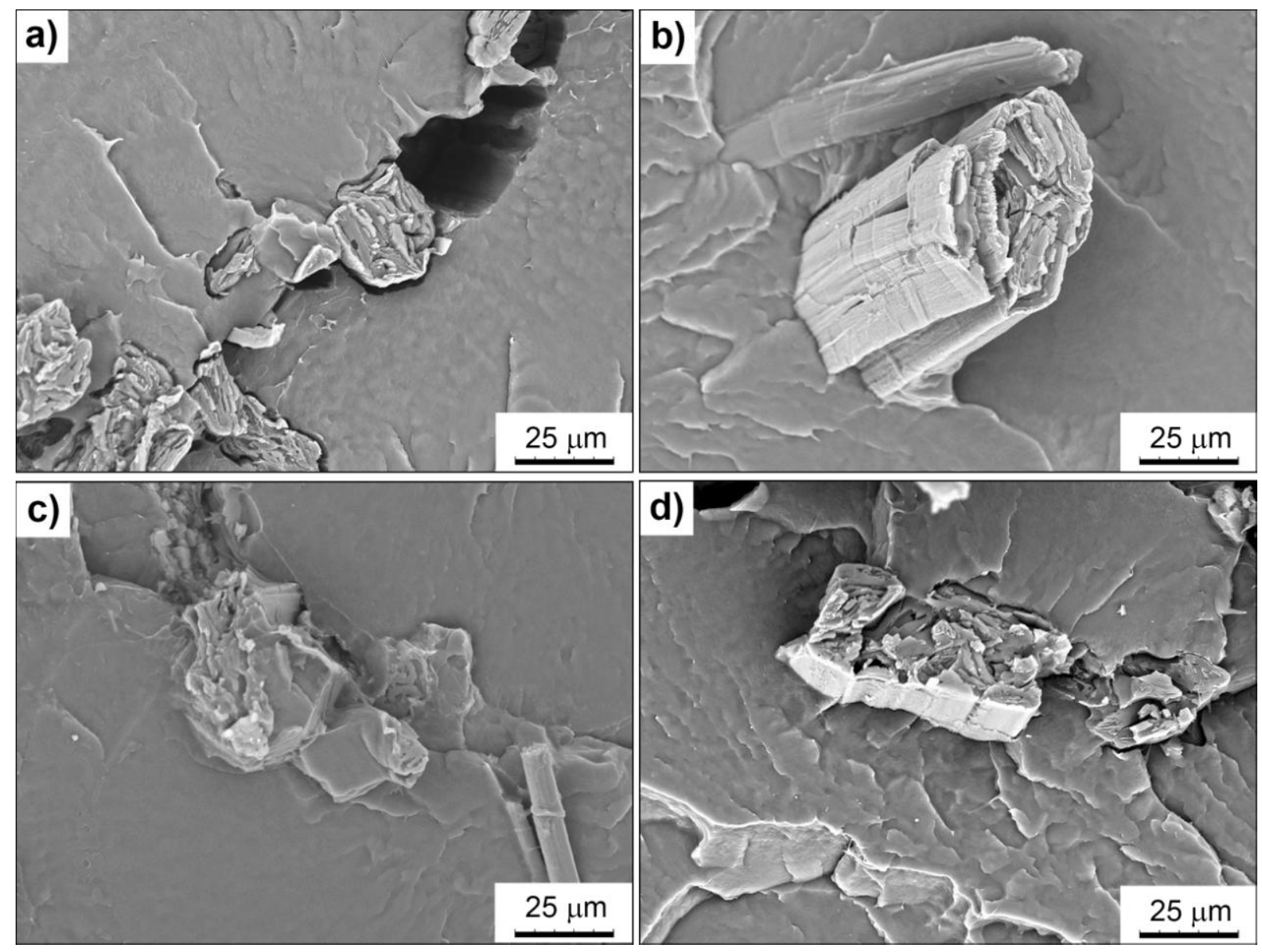

Fig. 10. SEM micrographs of preliminary PBAT-based composites containing < 5 wt.\% of: (a) uncut $\mathrm{HF}$, (b) $\mathrm{HF}_{\mathrm{Na}}$, (c) $\mathrm{HF}_{\mathrm{Na}}-\mathrm{npMCO}$, and (d) $\mathrm{HF}_{\mathrm{Na}}-\mathrm{C}$-pMCO fibres. (2-column fitting image)

444 Untreated HFs resulted very poorly adhered to the PBAT matrix (Fig. 10a). As shown in Fig. 10b, 445 while the pre-treatment with $\mathrm{NaOH}$ improved fibre/matrix adhesion. The adhesion of the fibres to 446 the PBAT matrix was noticeably further enhanced when $\mathrm{HF}_{\mathrm{Na}}-\mathrm{npMCO}$ (Fig. 10c) or $\mathrm{HF}_{\mathrm{Na}}-\mathrm{C}-$ 447 pMCOs (Fig. 10d) were used. No appreciable differences were evidenced between the two samples 448 containing the fibres modified with the MCOs. This suggests that the macrocycles purification step 449 and the subsequent addition of the catalyst prior to ED-ROP can be avoided, using directly the non450 purified macrocycles as the compatibilizing agent.

451 On the basis of these preliminary results, PBAT-based composites containing higher amounts 452 (about 40 wt.\%) of uncut oriented $\mathrm{HF}, \mathrm{HF}_{\mathrm{Na}}$, and $\mathrm{HF}_{\mathrm{Na}}-\mathrm{npMCO}$ fibres were prepared by 
453

compression moulding through the two-steps method described in the Materials and methods section.

The ensuing eco-composites were then characterized by SEM microscopy to estimate fibre-matrix interaction and by performing uniaxial tensile tests to evaluate their mechanical properties.

SEM micrographs of Fig. 11 confirm the higher interaction of MCO-modified fibres (Fig. 11 b,d) with the PBAT matrix if compared to the poor one shown by untreated HF fibres (Fig. 11 a,c).
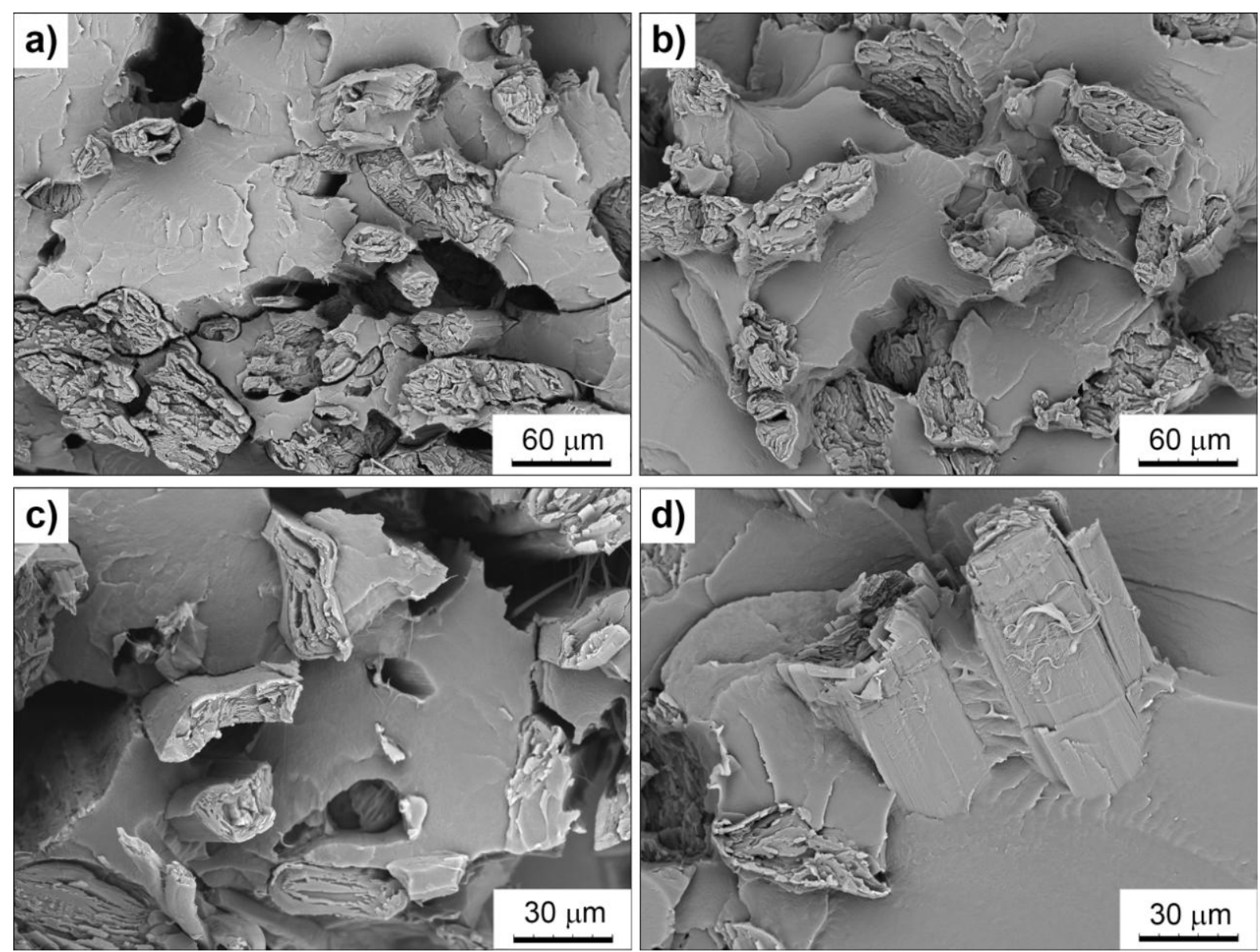

Fig. 11. SEM micrographs of PBAT-based composites containing: $(a, c)$ uncut $\mathrm{HF}$, and $(b, d) \mathrm{HF}_{\mathrm{Na}^{-}}$ npMCO fibres. (2-column fitting image)

Thermal stability of PBAT, HF fibres, and HF-PBAT composites was investigated by TGA analysis carried out in dynamic mode under nitrogen; the characteristic temperatures are reported in Table 4. A double step weight loss was observed in the TG curve of PBAT-based composites: the first step, between 300 and $400^{\circ} \mathrm{C}$, corresponds to the thermal degradation of the hemp fibres ( $T_{\text {onset }}$ and $\left.T_{V \max 1}\right)$, and the second one, in the range $400-500^{\circ} \mathrm{C}$, is ascribable to the thermal degradation of the PBAT matrix ( $T_{\text {onset } 2}$ and $T_{\operatorname{Vmax} 2}$ ). The degradation onset of the hemp fibres (about $340^{\circ} \mathrm{C}$ ) was shifted toward higher temperatures (about $360^{\circ} \mathrm{C}$ ) by their incorporation into the PBAT matrix. 
On the other hand, the thermal degradation of the PBAT $\left(T_{\text {onset } 2}\right)$ in the composites is practically independent on the fibre added and slightly lower than that of neat PBAT.

Table 4. $T_{\text {onset }}$ and $T_{V \max }$ decomposition temperatures of hemp fibres, neat PBAT, and PBAT/hemp fibres eco-composites determined by TGA at $10^{\circ} \mathrm{C} / \mathrm{min}$ under $\mathrm{N}_{2}$.

\begin{tabular}{|c|c|c|c|c|}
\hline Sample & $\begin{array}{c}\boldsymbol{T}_{\text {onset }} \\
\left({ }^{\circ} \mathbf{C}\right)\end{array}$ & $\begin{array}{c}\boldsymbol{T}_{\text {VmaxI }} \\
\left({ }^{\circ} \mathrm{C}\right)\end{array}$ & $\begin{array}{c}\boldsymbol{T}_{\text {onset } 2} \\
\left({ }^{\circ} \mathrm{C}\right)\end{array}$ & $\begin{array}{c}T_{V \max 2} \\
\left({ }^{\circ} \mathrm{C}\right)\end{array}$ \\
\hline PBAT & - & - & 385 & 418 \\
\hline PBAT + HF & 361 & 384 & 372 & 422 \\
\hline $\mathbf{P B A T}+\mathbf{H F}_{\mathrm{Na}}$ & 353 & 378 & 375 & 425 \\
\hline $\mathrm{PBAT}+\mathrm{HF}_{\mathrm{Na}}-\mathrm{npMCO}$ & 357 & 384 & 362 & 419 \\
\hline
\end{tabular}

476

Table 5. Tensile modulus $(E)$, stress $\left(\sigma_{b}\right)$ and strain $\left(\varepsilon_{b}\right)$ at break obtained for neat PBAT and PBAT-based composites containing about $40 \mathrm{wt} . \%$ hemp fibres.

\begin{tabular}{l|c|c|c}
\multicolumn{1}{c|}{ Sample } & $\begin{array}{c}\boldsymbol{E} \\
(\mathbf{M P a})\end{array}$ & $\begin{array}{c}\boldsymbol{\sigma}_{\boldsymbol{b}} \\
(\mathbf{M P a})\end{array}$ & $\begin{array}{c}\boldsymbol{\varepsilon}_{\boldsymbol{b}} \\
(\boldsymbol{\%})\end{array}$ \\
\hline PBAT & $75 \pm 8$ & $24 \pm 2$ & $1527 \pm 86$ \\
\hline PBAT + HF & $2435 \pm 249$ & $111 \pm 14$ & $12 \pm 1$ \\
\hline PBAT + HF & $4805 \pm 550$ & $97 \pm 12$ & $4 \pm 1$ \\
\hline PBAT + HF & $5480 \pm 280$ & $76 \pm 14$ & $2 \pm 0.3$
\end{tabular}

Among the composites, the sample containing the MCO-modified fibres (PBAT $+\mathrm{HF}_{\mathrm{Na}}-\mathrm{npMCO}$ ) revealed the highest $E$ value, indicative of the beneficial effect of such modification on the fibrematrix adhesion, which in turn promotes under loading an efficient stress transfer from matrix to the fibrous reinforcement. The slight decrease of the stress at break could be ascribed to a mechanical weakening of the hemp fibres due to the thermal treatment carried out during the ED-ROP reaction $\left(190^{\circ} \mathrm{C}, 30 \mathrm{~min}\right)$. Even though a TGA isothermal analysis carried out at $200^{\circ} \mathrm{C}$ in $\mathrm{O}_{2}$ atmosphere for 
30 min revealed a good thermo-oxidative resistance of $\mathrm{HF}_{\mathrm{Na}} \mathrm{s}$ fibres (the weight loss is about $0.5 \%$ ), the maintenance at high temperature in oxidative atmosphere could be slightly detrimental to the mechanical properties of the fibres, as reported by Prasad et al. [34].

Dynamic-mechanical analysis (DMA) of PBAT and PBAT-based composites containing $\mathrm{HF}_{\mathrm{Na}}$ and $\mathrm{HF}_{\mathrm{Na}}-\mathrm{npMCO}$ fibres was carried out to evaluate the effective reinforcement exerted by the fibres in terms of elastic or storage modulus $\left(G^{\prime}\right)$. The resulting curves are shown in Fig. 12a. The presence of the long fibres in the composites resulted in a strong increase of the mechanical properties with respect to those of the neat $\operatorname{PBAT}\left(\mathrm{G}^{\prime}\right.$ at $\left.1 \mathrm{~Hz}=47 \mathrm{MPa}\right)$. As expected, this effect is more pronounced along the fibre axis than in the orthogonal direction: in fact, the values of G' measured at $1 \mathrm{~Hz}$ are respectively 510 and $260 \mathrm{MPa}$ for the $\mathrm{PBAT}+\mathrm{HF}_{\mathrm{Na}}$ composite, and the corresponding values for $\mathrm{PBAT}+\mathrm{HF}_{\mathrm{Na}}-\mathrm{npMCO}$ are 770 and $320 \mathrm{MPa}$. The beneficial effect of the MCOfunctionalization of HFs with respect to the simple alkali treatment is evidenced by an increase in G' of the $51 \%$ in the longitudinal direction and $23 \%$ in the transversal one.

The effectiveness of the compatibilization through MCOs is also visible in the FE-SEM micrograph of the PBAT $+\mathrm{HF}_{\mathrm{Na}}-\mathrm{npMCO}$ sample taken after DMA analysis and reported in Fig. 12b. Besides fibres detached from the matrix, as a consequence of the torsional strain, the fibre external layer remain adhered to the polymer, indicating the good adhesion between the HF-modified surface and the PBAT matrix.

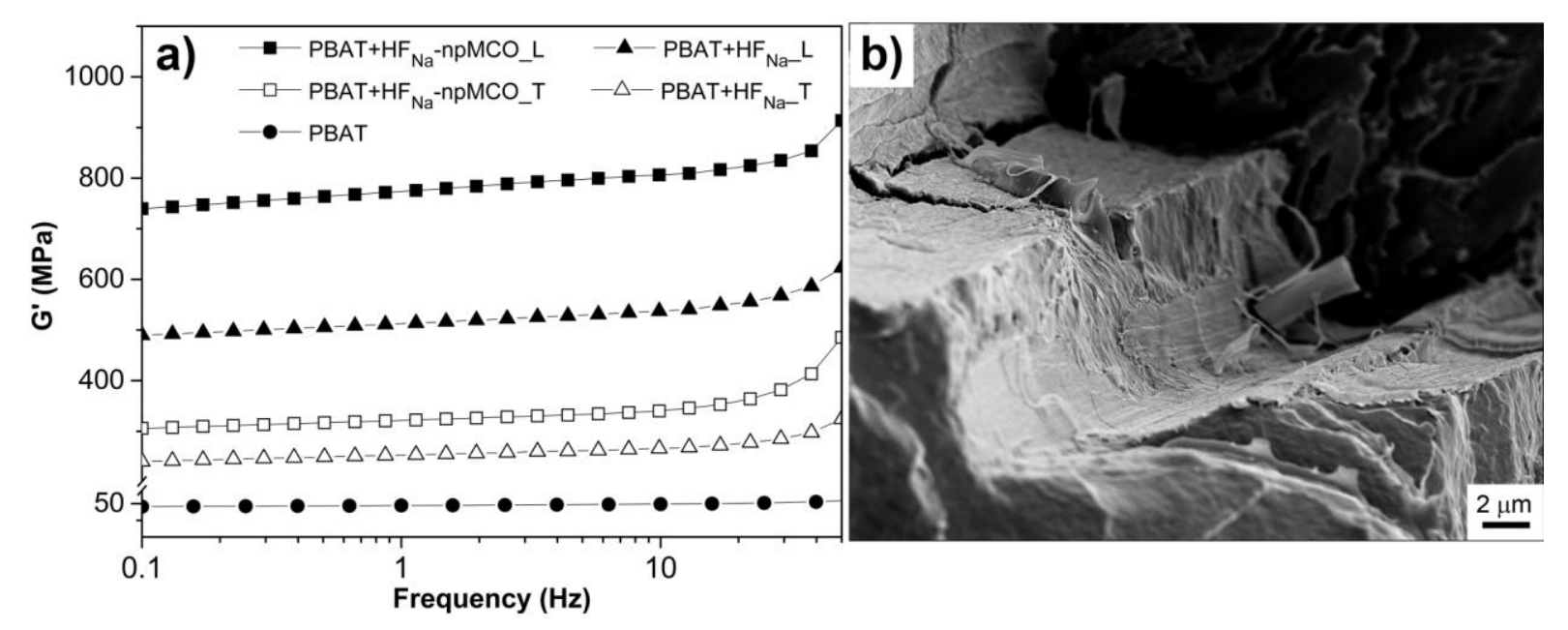

Fig. 12. (a) DMA curves of PBAT, PBAT $+\mathrm{HF}_{\mathrm{Na}}$ and PBAT $+\mathrm{HF}_{\mathrm{Na}}-\mathrm{npMCO}$ samples obtained in longitudinal (L) and transversal (T) directions; (b) FE-SEM micrograph of PBAT $+\mathrm{HF}_{\mathrm{Na}}-\mathrm{npMCO}$ sample after DMA analysis. (2-column fitting image) 


\section{Conclusions}

520 CDP of poly(1,4-butylene adipate-co-terephthalate) (PBAT) was, for the first time, successfully 521 performed at high dilution, in mild conditions and in the presence of a suitable transesterification 522 catalyst, achieving quantitative yields of the corresponding macrocyclic oligomers (MCOs).

523 The reconversion of the MCOs into linear polymeric chains was successfully accomplished by ED524 ROP, carried out by simply heating the neat macrocycles as obtained from CDP, without any 525 further addition of catalyst.

526 ED-ROP of the MCOs adsorbed onto hemp fibres previously mildly treated with acqueous $\mathrm{NaOH}$ 527 to remove non-cellulosic and amorphous components and favour accessibility to the functional 528 groups, led to modified fibres with anchored PBAT oligomers, that is to a perfectly suited 529 compatibilizing agent for PBAT composites reinforced with hemp fibres.

530 PBAT-based composites containing about 40 wt.\% of pristine, NaOH-treated, and MCO-modified 531 hemp fibres were prepared by compression moulding. The sample compatibilized with the MCO532 modified fibres showed the best fibre/matrix adhesion, which resulted in enhanced mechanical 533 properties in terms of elastic modulus with respect to not only the neat PBAT but also to the other 534 composites.

535 The overall results gathered so far validate the compatibilization strategy adopted for preparing eco536 friendly composite materials based on a biodegradable polyester matrix reinforced with high 537 amounts of low cost fibres arising from hemp manufacturing by-products.

\section{Acknowledgements}

541 Dr. F. Giunco (CNR-ISMAC Genova) for helpful contribution, Mr. M. Canetti (CNR-ISMAC Milano) for WAXD measurements.

This research did not receive any specific grant from funding agencies in the public, commercial, or not-for-profit sectors.

\section{References}

549 [1] P. Hodge, Entropically driven ring-opening polymerization of strainless organic macrocycles, 550 Chem. Rev. 114 (2014) 2278-2312.

551 [2] P. Hodge, Recycling of condensation polymers via ring-chain equilibria, Polym. Adv. Technol. 55226 (2015) 797-803. 
553 [3] L. Conzatti, R. Utzeri, P. Hodge, P. Stagnaro, A novel tin-based imidazolium-modified 554 montmorillonite catalyst for the preparation of poly(butylene terephthalate)-based nanocomposites 555 using in situ entropically-driven ring-opening polymerization, RSC Adv. 5 (2015) 6222-6231.

556 [4] P. Hodge, Cyclodepolymerization as a method for the synthesis of macrocyclic oligomers, 557 React. Funct. Polym. 80 (2014) 21-32.

558 [5] D.J. Brunelle, Macrocycles for the synthesis of high molecular weight polymers, in: J.R. Ebdon, 559 G.C. Eastmond (Eds.), New methods of polymer synthesis - vol. 2, Blackie, London, 1995, pp. 197560235.

561 [6] A. Ben-Haida, H.M. Colquhoun, P. Hodge, J.L. Stanford, A novel approach to processing high562 performance polymers that exploits entropically-driven ring-opening polymerization, Macromol. 563 Rapid Comm. 26 (2005) 1377-1382.

564 [7] M. Alessi, L. Conzatti, P. Hodge, S. Taglialatela Scafati, P. Stagnaro, A possible means to assist 565 the processing of PET, PTT and PBT, Macromol. Mater. Eng. 295 (2010) 374-380.

566 [8] M.N. Belgacem, A. Gandini, Monomers, polymers and composites from renewable resources, 567 Elsevier, Oxford UK, 2008.

568 [9] O. Faruk, A.K. Bledzki, H.P. Fink, M. Sain, Biocomposites reinforced with natural fibers: 2000569 2010, Prog. Polym. Sci. 37 (2012) 1552-1596.

570 [10] M.P.M. Dicker, A.B. Baker, P.F. Duckworth, A.B. Baker, G. Francois, M.K. Hazzard, P.M. 571 Weaver, Green composites: A review of material attributes and complementary applications, 572 Compos. Part A-Appl. S. 56 (2014) 280-289.

573 [11] T. Gurunathan, S. Mohanty, S. K. Nayak, A review of the recent developments in 574 biocomposites based on natural fibres and their application perspectives, Compos. Part A-Appl. S., $57577(2015) 1-25$.

576 [12] K. L. Pickering, M. G. Aruan Efendy, T. M. Le, A review of recent developments in natural 577 fibre composites and their mechanical performance, Compos. Part A-Appl. S. 83 (2016) 98-112.

578 [13] S. Kalia, B.S. Kaith, I. Kaur, Pretreatments of natural fibers and their application as reinforcing 579 material in polymer composites - A review, Polym. Eng. Sci., 49 (2009) 1253-1272.

580 [14] L. Conzatti, F. Giunco, P. Stagnaro, M. Capobianco, M. Castellano, E. Marsano, Polyester581 based biocomposites containing wool fibres, Compos. Part A-Appl. S. 43 (2012) 1113-1119.

582 [15] L. Conzatti, F. Giunco, P. Stagnaro, A. Patrucco, C. Marano, M. Rink, E. Marsano, 583 Composites based on polypropylene and short wool fibres, Compos. Part A-Appl. S. 47 (2013) 165584171. 
[16] L. Conzatti, F. Giunco, P. Stagnaro, A. Patrucco, C. Tonin, C. Marano, M. Rink, E. Marsano,

586 Wool fibres functionalised with a silane-based coupling agent for reinforced polypropylene composites, Compos. Part A-Appl. S. 61 (2014) 51-59.

[17] L. Conzatti, R. Utzeri, P. Hodge, P. Stagnaro, Biodegradable polyester-based eco-composites containing hemp fibers modified with macrocyclic oligomers, AIP Conference Proceedings 1736,

591 [18] L.Y. Mwaikambo, M.P. Ansell, Mechanical properties of alkali treated plant fibres and their 592 potential as reinforcement materials. I. hemp fibres, J Mater. Sci. 41 (2006) 2483-2496.

593 [19] L.E. Hult, T. Iversen, J. Sugiyama, Characterization of the supramolecular structure of 594 cellulose in wood pulp fibres, Cellulose 10 (2003) 103-110.

[20] C.J. Garvey, I.H. Parker, G.P. Simon,On the interpretation of X-ray diffraction powder patterns in terms of the nanostructure of cellulose I fibres. Macromol. Chem. Phys. 206 (2005) 1568-1575. [21] S.D. Kamau, P. Hodge, M. Helliwell, Cyclo-depolymerization of poly(propylene terephthalate): Some ring-opening polymerizations of the cyclic oligomers produced, Polym. Adv. 599 Technol. 14 (2003) 492-501.

600 [22] X. Li, L.G. Tabil, S. Panigrahi, Chemical treatments of natural fiber for use in natural fiber601 reinforced composites: A review, J. Polym. Environ. 15 (2007) 25-33.

602 [23] R. Agrawal, N.S. Saxena, K.B. Sharma, S. Thomas, M.S. Sreekala, Activation energy and 603 crystallization kinetics of untreated and treated oil palm fibre reinforced phenol formaldehyde 604 composites, Mat. Sci. Eng. A 277 (2000) 77-82.

605 [24] T. Fisher, M. Hajaligol, B. Waymack, D. Kellogg, Pyrolysis behavior and kinetics of biomass 606 derived materials, J. Anal. Appl. Pyrol. 62 (2002) 331-349.

607 [25] C.M. Popescu, M.C. Popescu, C. Vasile, Structural analysis of photodegraded lime wood by 608 means of FT-IR and 2D IR correlation spectroscopy, Int. J. Biolog. Macromol., 48 (2011) 667-675.

609 [26] K.K. Pandey, A study of chemical structure of soft and hardwood and wood polymers by FTIR 610 spectroscopy, J. Appl. Polym. Sci. 71 (1999) 1969-1975.

611 [27] K. Bilba, M.A. Arsene, A. Ouensanga, Study of banana and coconut fibers - Botanical 612 composition, thermal degradation and textural observations, Biores. Technol. 98 (2007) 58-68.

613 [28] C.J. Garvey, I.H. Parker, G.P. Simon, On the interpretation of X-ray diffraction powder 614 patterns in terms of the nanostructure of cellulose I fibres, Macromol. Chem. Phys. 206 (2005) $615 \quad 1568-1575$.

616 [29] S. Park, J.O. Baker, M.E. Himmel, P.A. Parilla, D.K. Johnson, Cellulose crystallinity index: 617 measurement techniques and their impact on interpreting cellulase performance, Biotechnol. 618 Biofuels, 3 (2010). 
619 [30] S. Ouajai, R.A., Shanks, Composition, structure and thermal degradation of hemp cellulose 620 after chemical treatments, Polym. Degrad. Stab. 89 (2005) 327-335.

621 [31] G.W. Beckermann, K.L. Pickering, Engineering and evaluation of hemp fibre reinforced 622 polypropylene composites: Fibre treatment and matrix modification, Composites: Part A 39 (2008) 623 979-988.

624 [32] M.A. Sawpan, K.L. Pickering, A. Fernyhough, Effect of various chemical treatments on the 625 fibre structure and tensile properties of industrial hemp fibres, Composites: Part A 42 (2011) 888626895.

627 [33] V. Tserki, N.E. Zafeiropoulos, F. Simon, C. Panayiotou, A study of the effect of acetylation 628 and propionylation surface treatments on natural fibres, Compos. Part A-Appl. S. 36 (2005) 11106291118.

630 [34] B. M. Prasad, M. M. Sain, Mechanical properties of thermally treated hemp fibers in inert 631 atmosphere for potential composite reinforcement, Mat. Res. Innovat. 7 (2003) 231-238. 


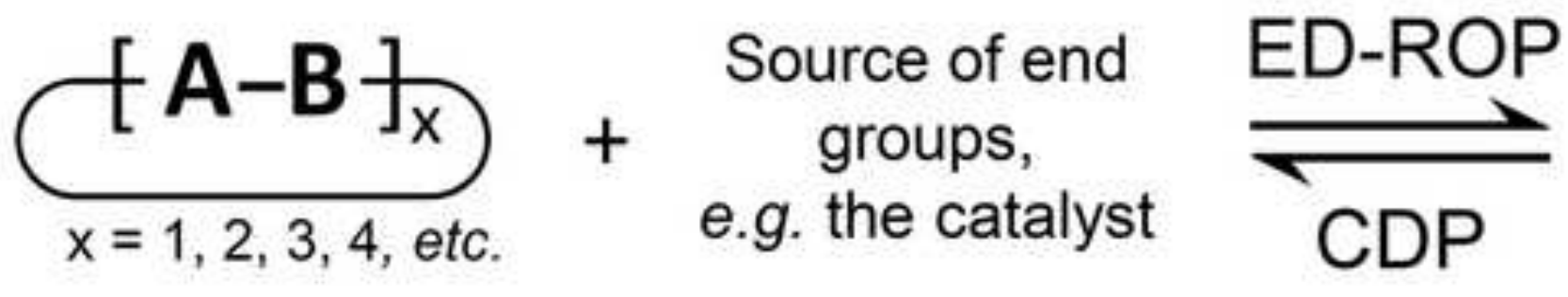

MCOs

FAVOURED BY HIGH DILUTION

\section{$\mathbf{f}-\mathbf{B}\}_{n}$}

Linear polymer

FAVOURED BY

$\mathrm{HIGH}$

CONCENTRATION 


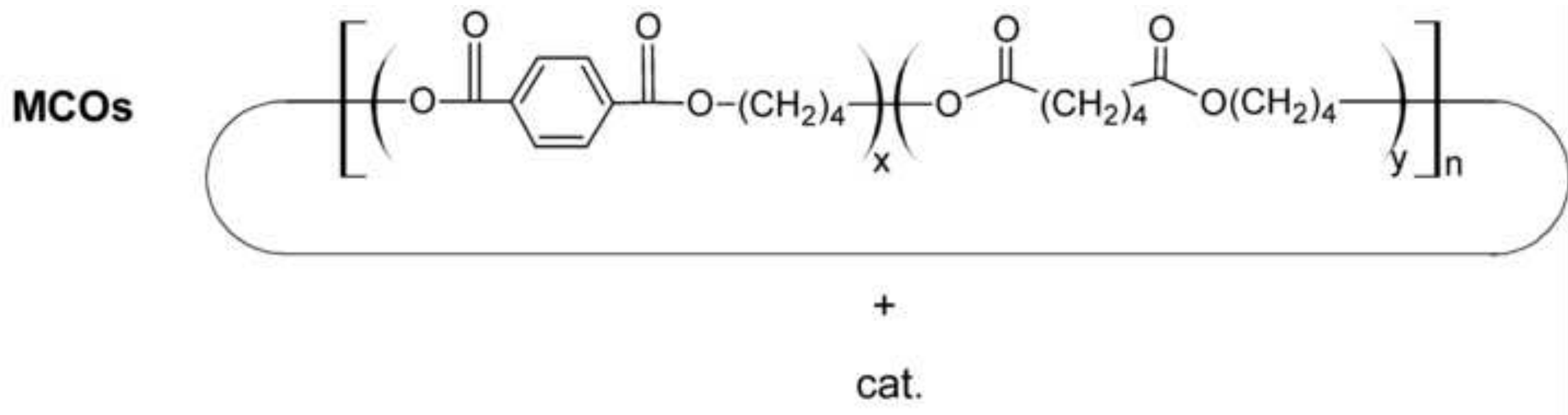

PBAT

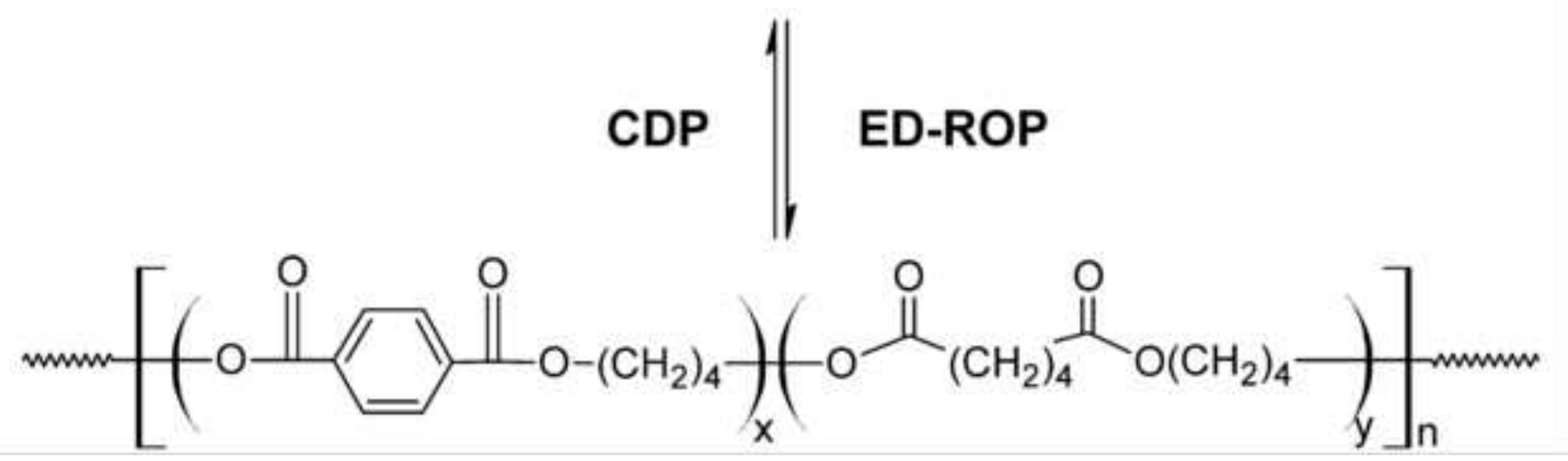




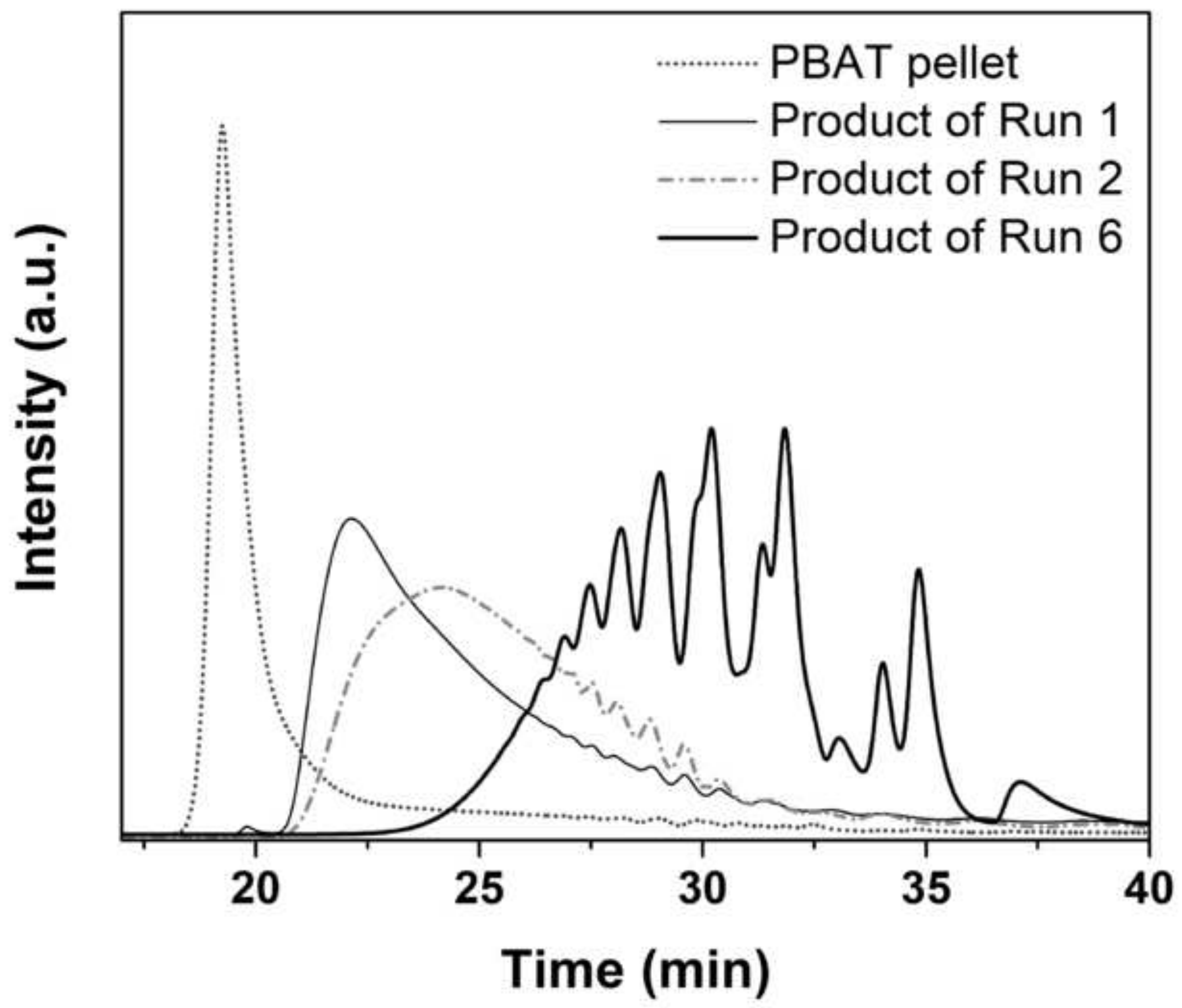




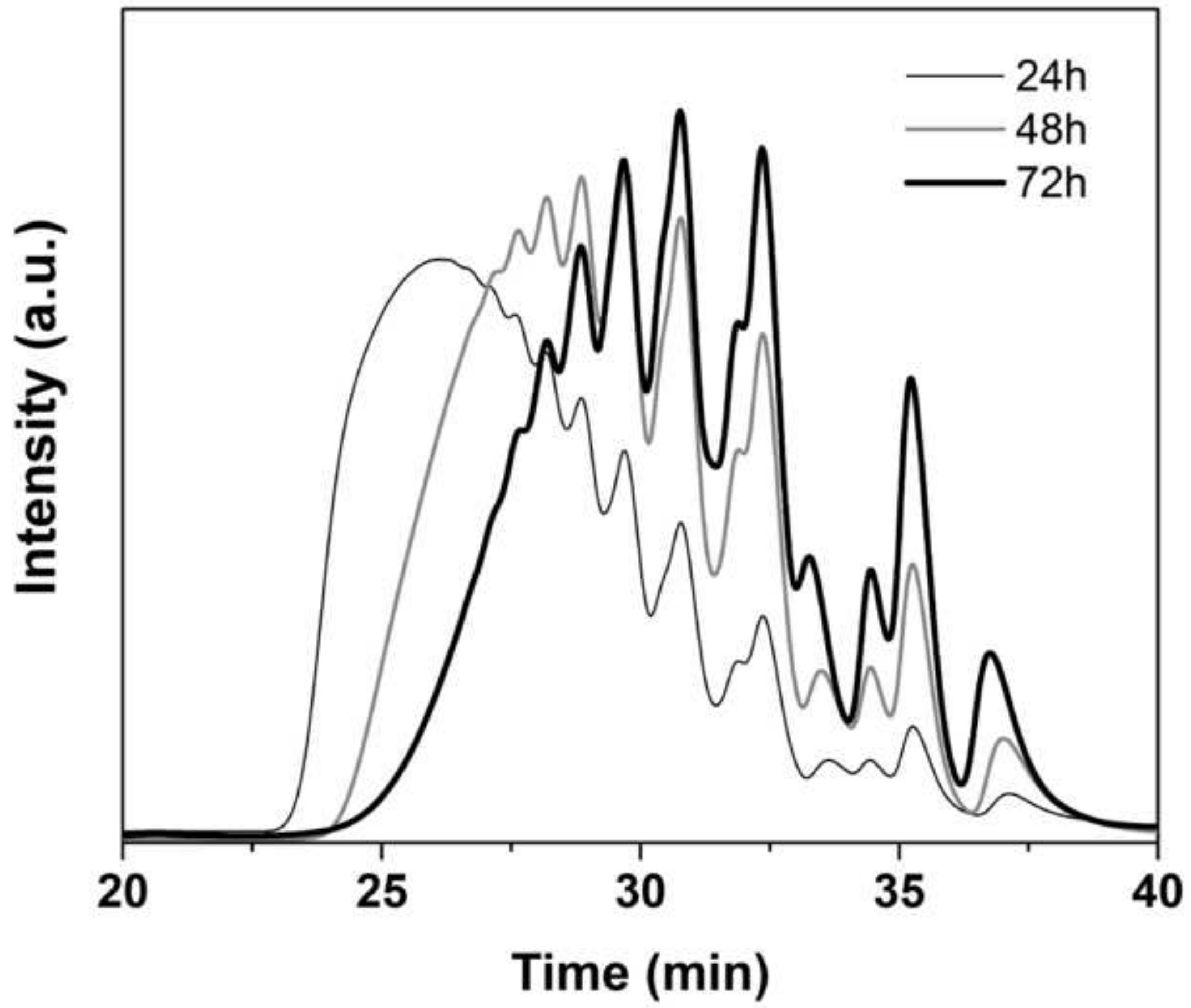




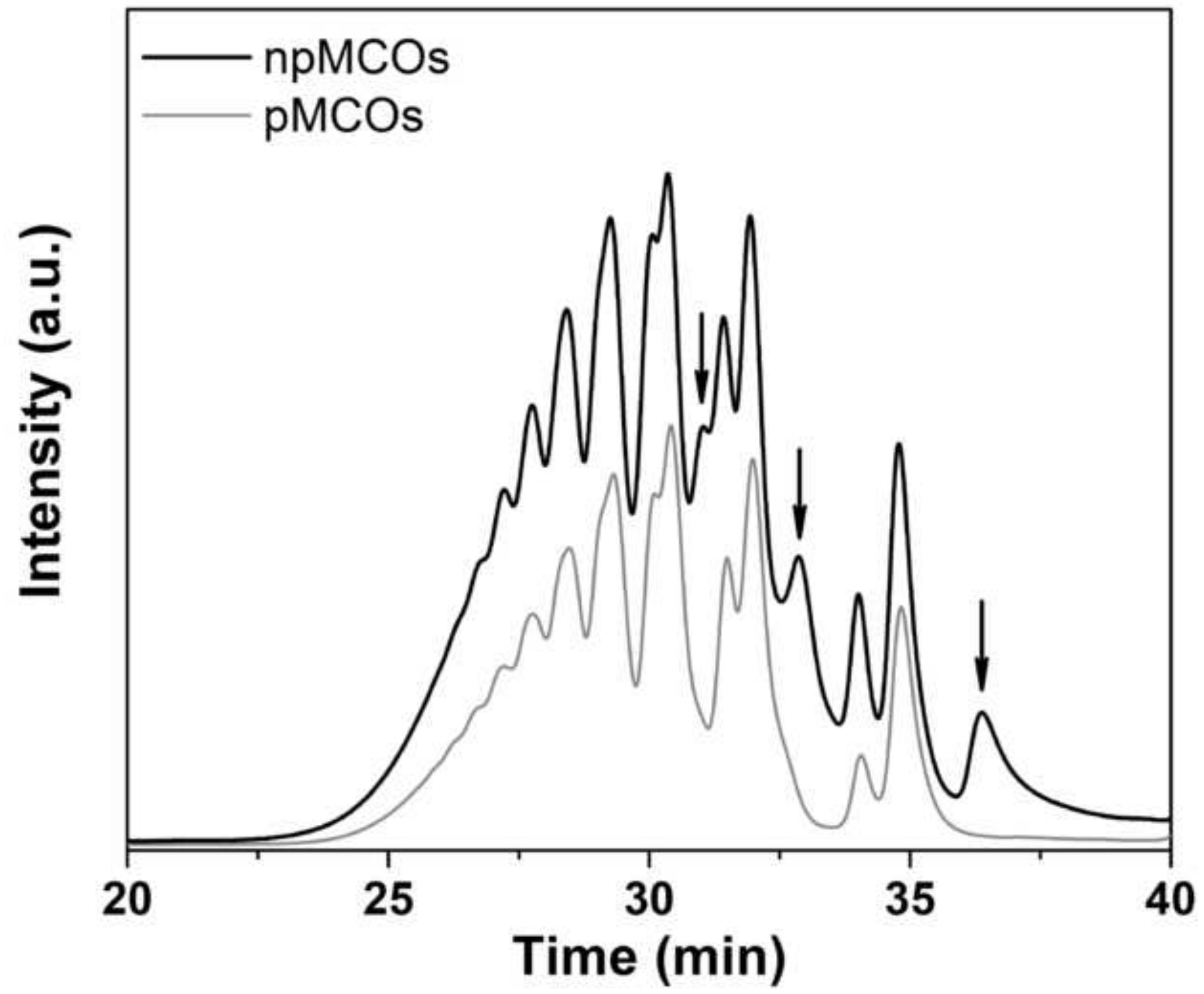



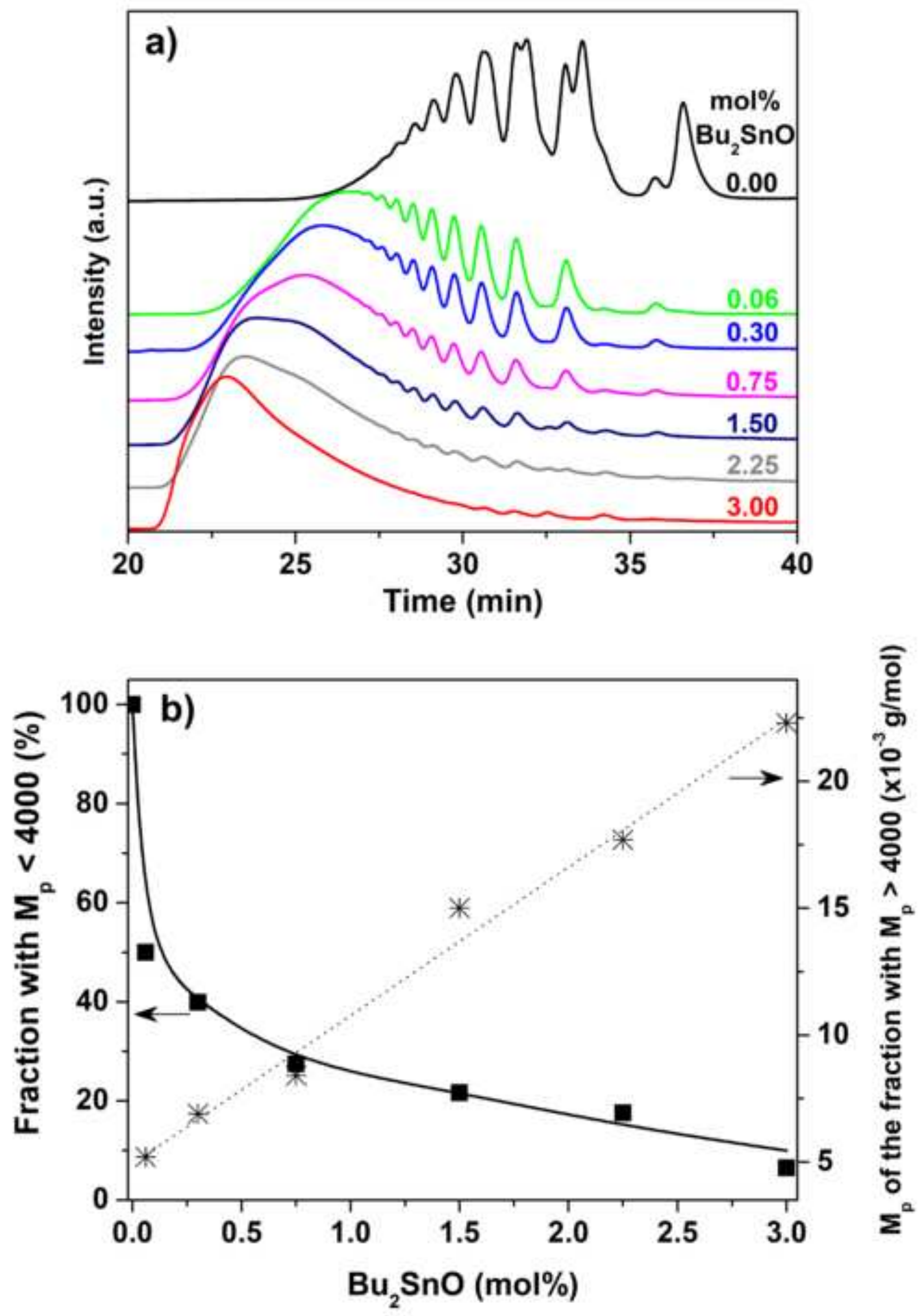

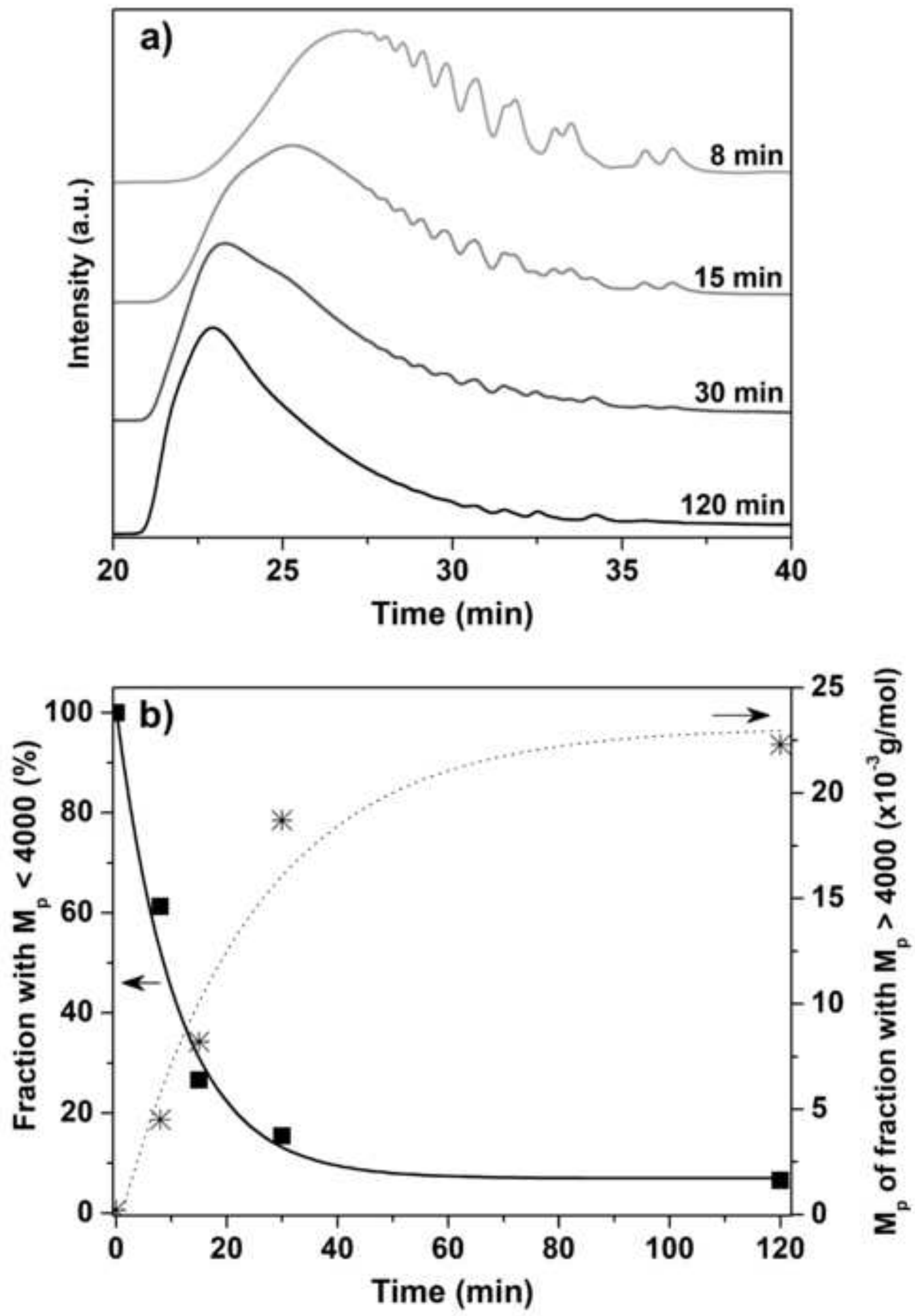

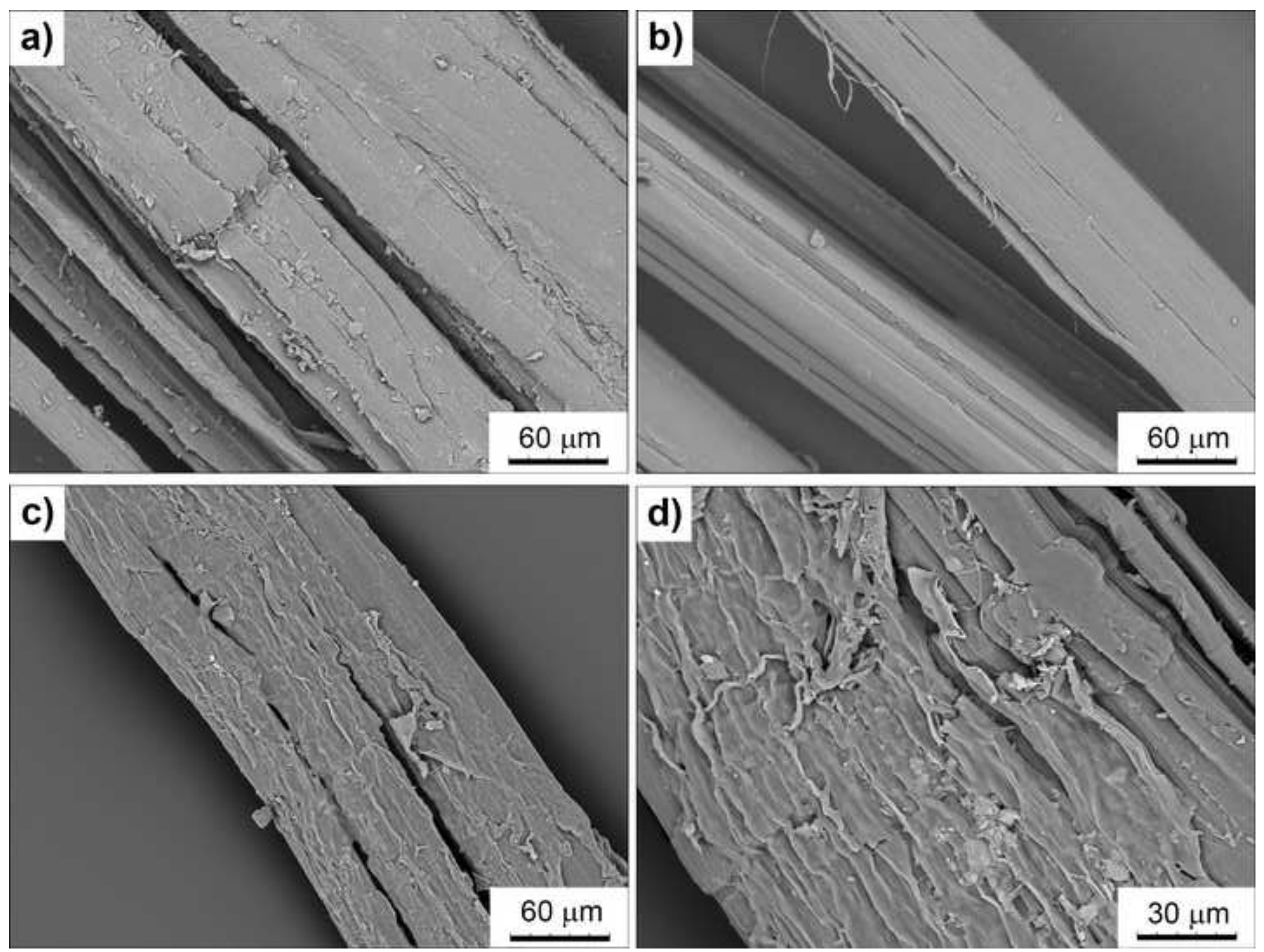


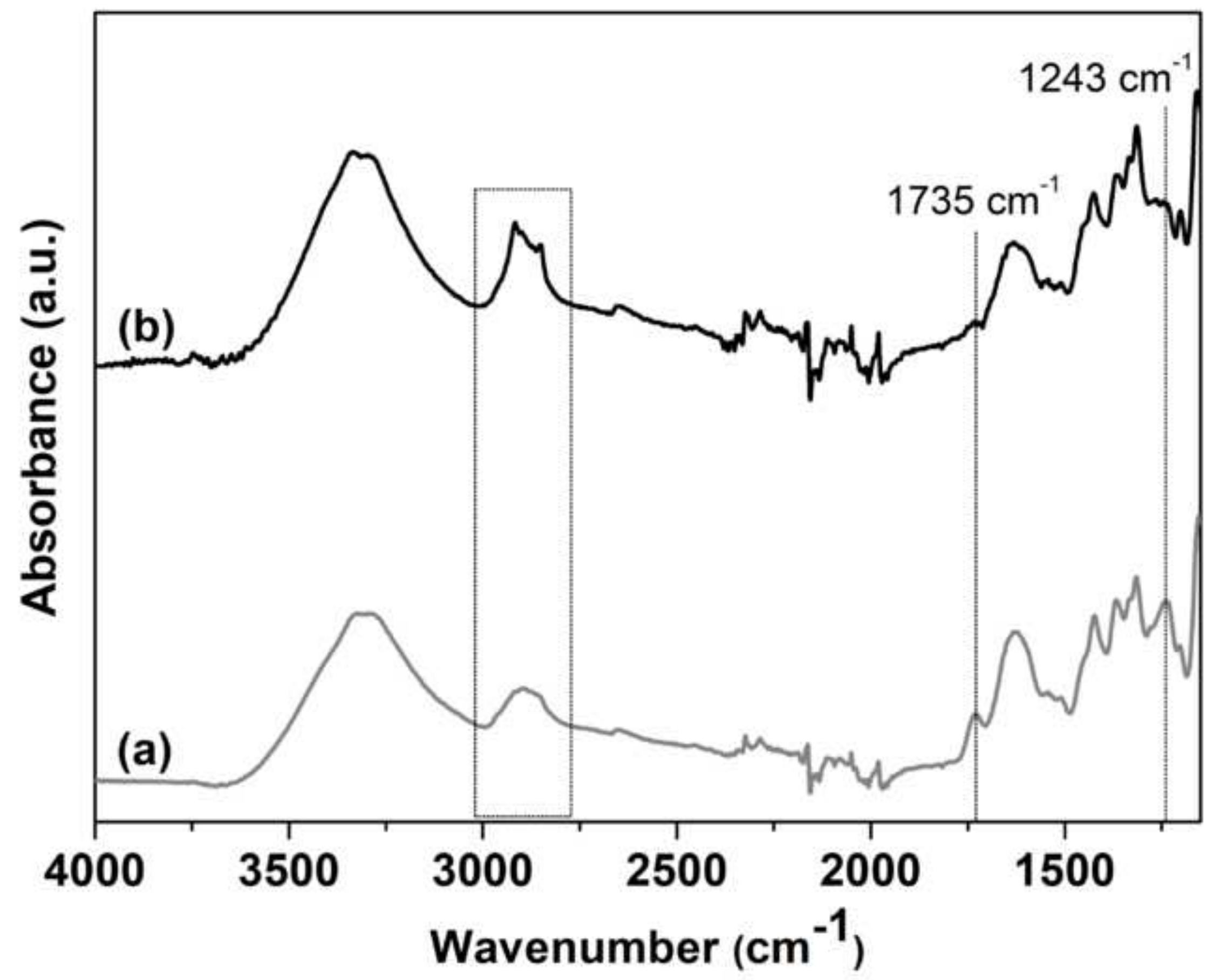


Figure 8
Click here to download high resolution image
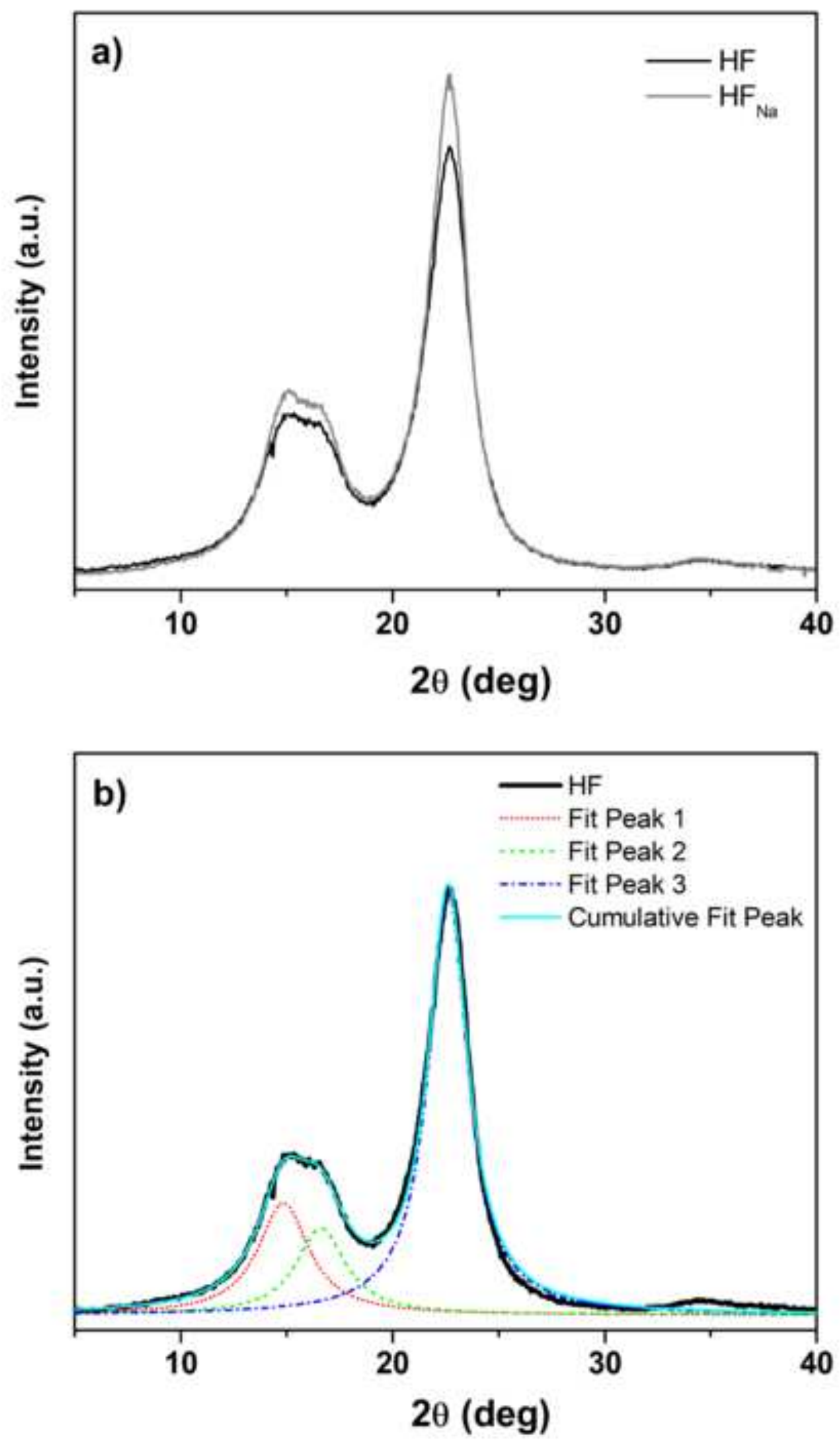


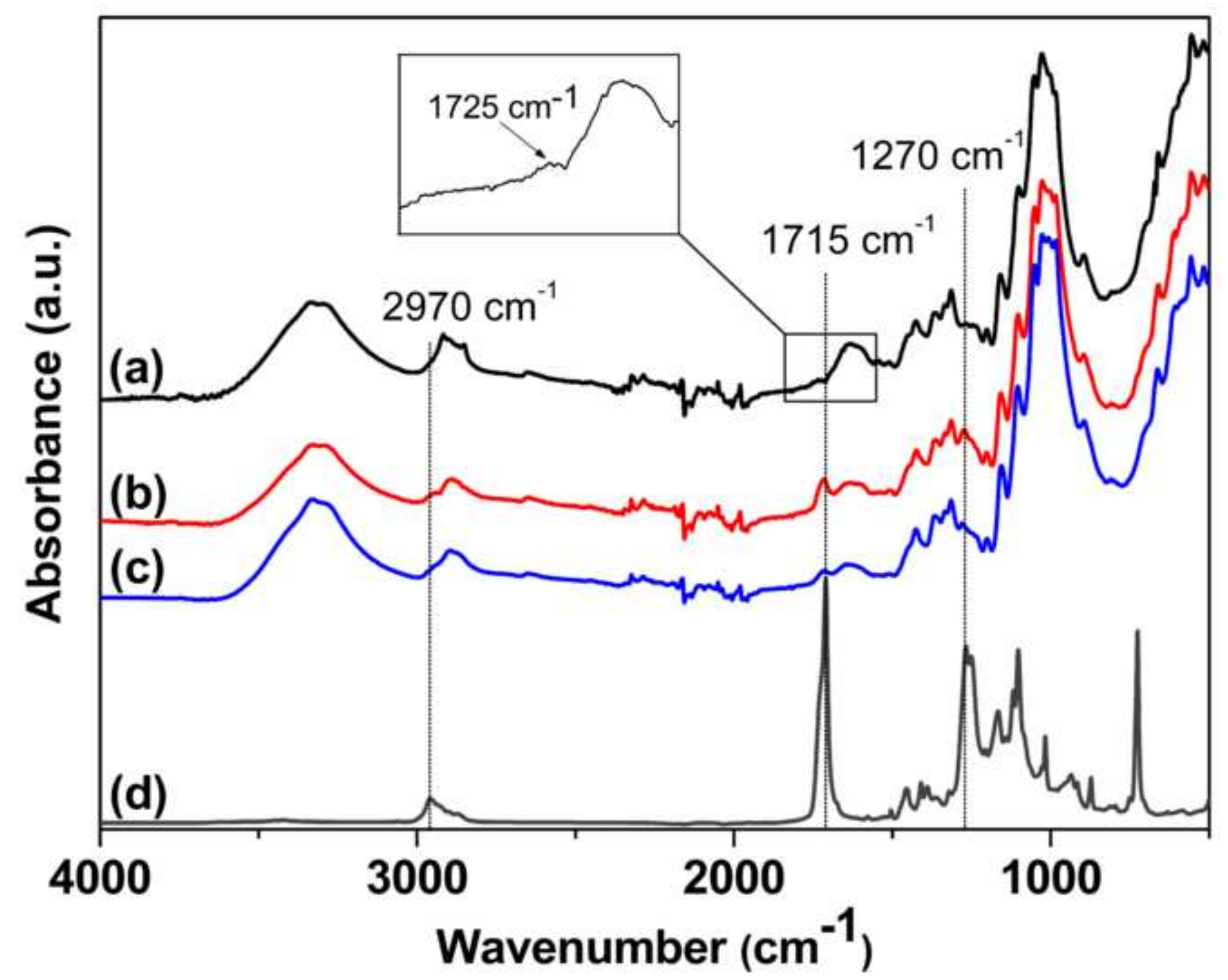


Figure 10
to download high resolution imag
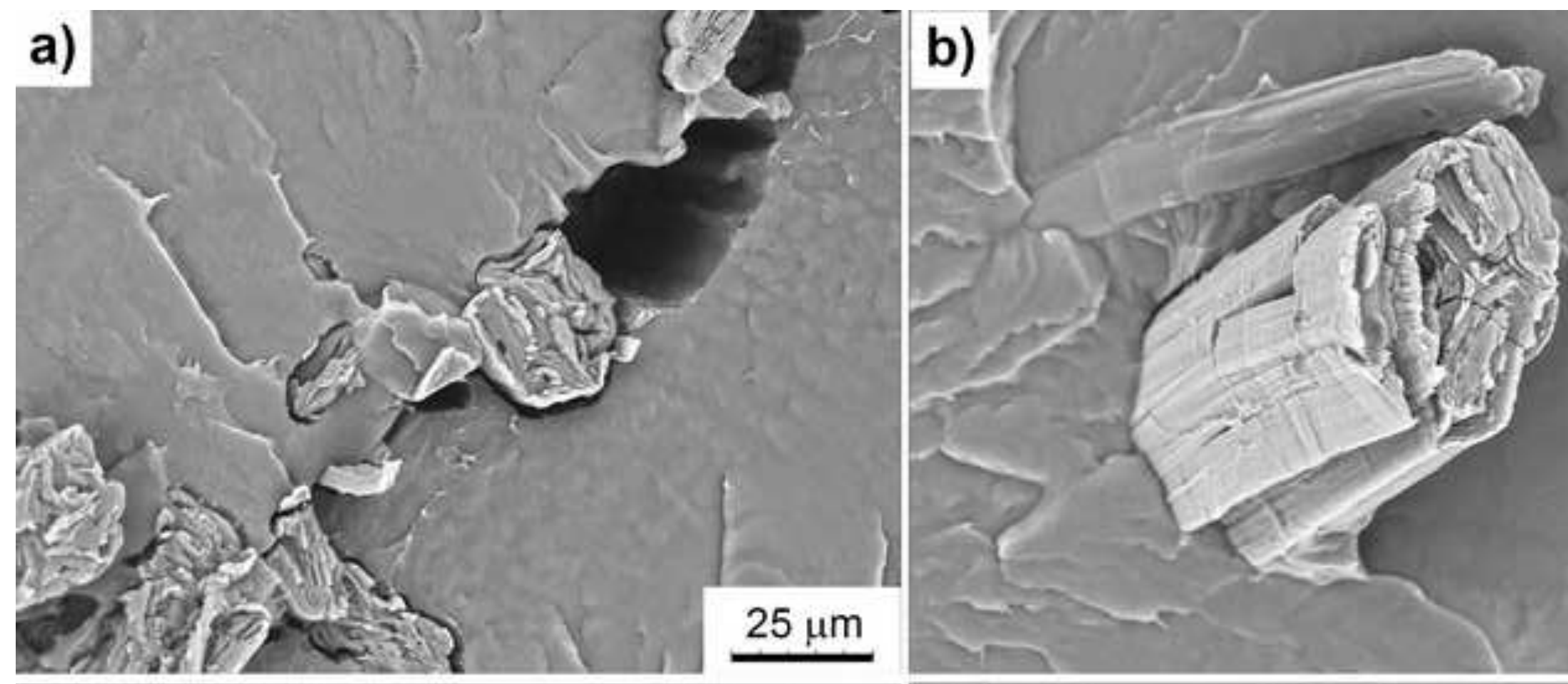

$25 \mu \mathrm{m}$

c)
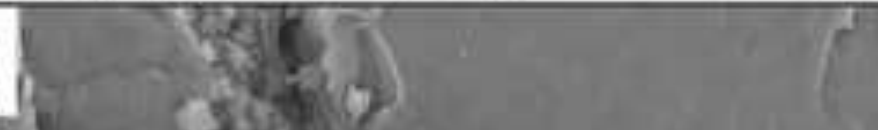

d)
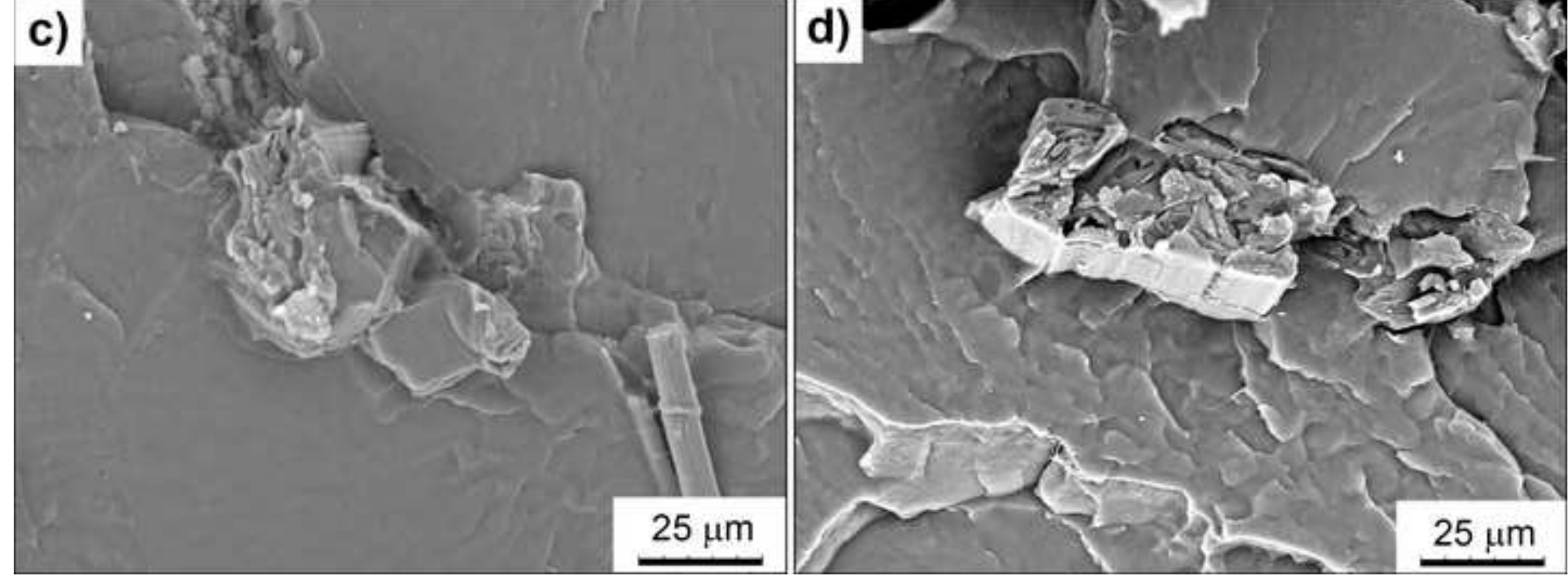

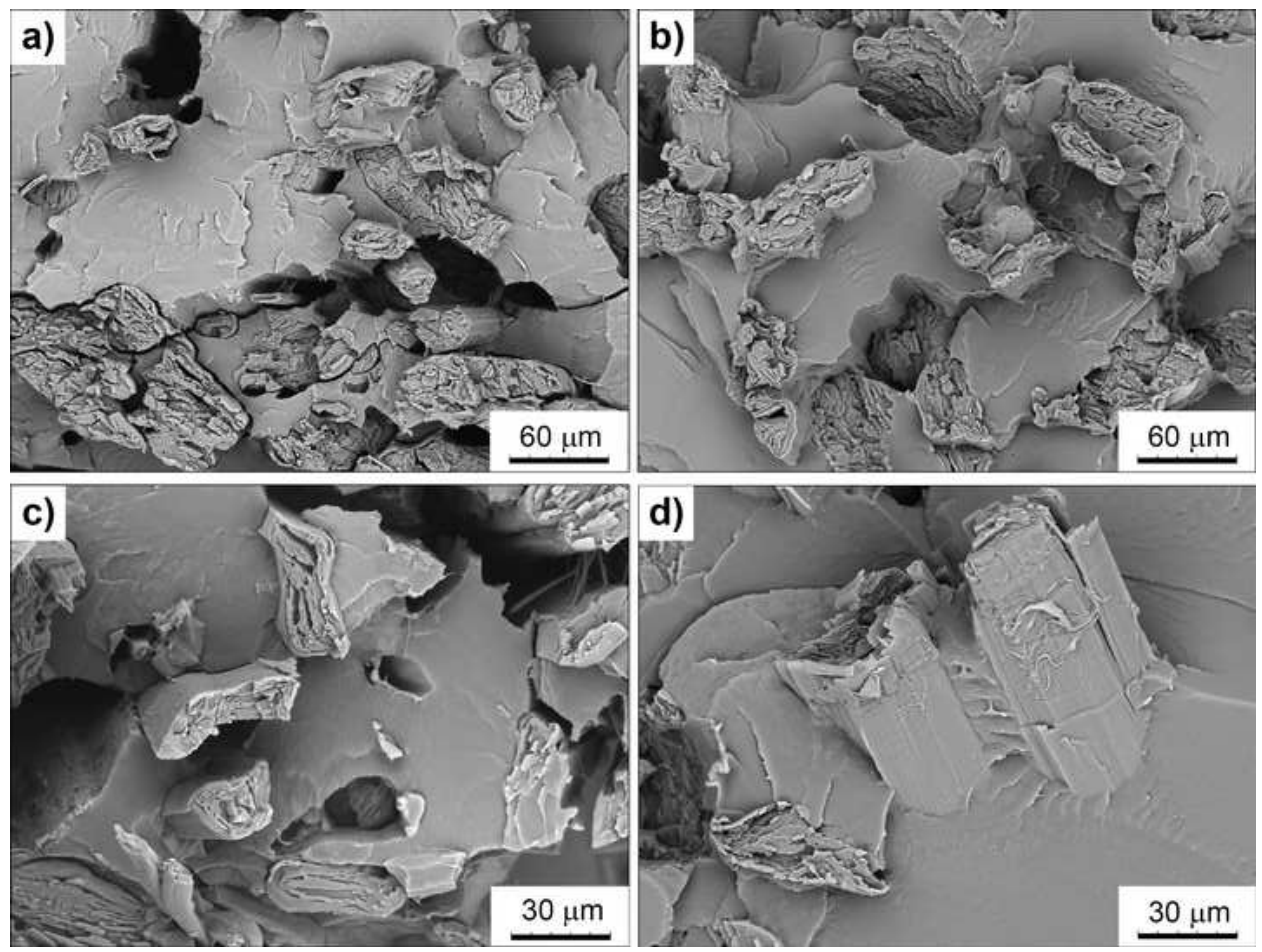
Click here to download high resolution image

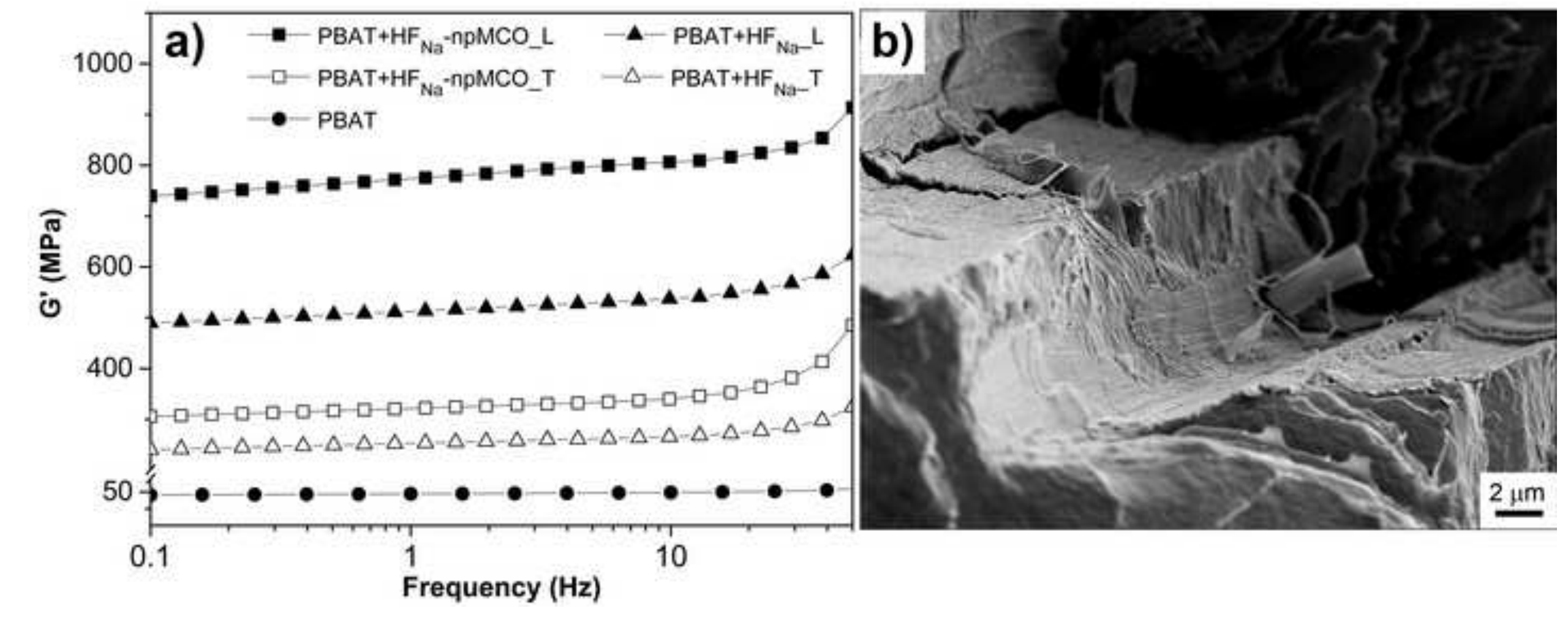




\section{Highlights}

- Macrocyclic oligomers (MCOs) easily obtained by cyclo-depolymerization of PBAT polyester

- The MCOs easily underwent entropically driven ring opening polymerization (ED-ROP)

- Low cost hemp fibres efficiently modified with the MCOs via ED-ROP

- Good fibre/matrix adhesion in PBAT composites containing MCO-modified hemp fibres

- Good mechanical performance of the PBAT/modified hemp fibres biodegradable eco-composites 